\title{
Identification of structures and mechanisms in a flow field by POD analysis for input data obtained from visualization and PIV
}

\author{
Rut Vitkovicova ${ }^{1}\left[\right.$ ] Yoshifumi Yokoi $^{2} \cdot$ Tomas Hyhlik $^{1}$
}

Received: 6 September 2019 / Revised: 28 June 2020 / Accepted: 1 July 2020 / Published online: 9 July 2020

(c) The Author(s) 2020

\begin{abstract}
This paper investigates the application of proper orthogonal decomposition (POD) for data obtained from visualizations. Using the POD method, the flow field behind one and two cylinders in a staggered configuration was analyzed. The data processed by this method were obtained from experimental measurements of flow fields using the particle image velocimetry (PIV) method and visualization. The dominant frequencies of the flow pattern from these data were compared using constant temperature anemometry (CTA) measurements. Attention was mainly focused on the flow at three Reynolds numbers: 500, 1200 , and 2500. Velocity and vortex fields were created from PIV measurements in the wind tunnel for $R e=500$, and video images of flow fields were obtained from dye visualizations in the hydrodynamic tunnel. The components of velocity, vorticity (both of PIV), and change in grayscale (from visualization) were used as input data for POD analysis. A methodology for data processing from visualizations was developed for subsequent analysis using the POD method. A new technique has been found to identify structures in the wake of the cylinders in a staggered configuration by analyzing POD based on various types of input data. The measured fields of dominant frequencies from the CTA and a thorough analysis of the POD modes and their relative energy values for each type of data made it possible to identify the structures and mechanisms that occur in the wake of cylinders. This analysis facilitated a better understanding of the importance of these structures and mechanisms, which can then be used to control the flow behind the cylinders.
\end{abstract}

Rut Vitkovicova

rut.vitkovicova@fs.cvut.cz; rut.vitkovicova@gmail.com

1 Faculty of Mechanical Engineering, Czech Technical University in Prague, Technicka 4, 16607 Prague 6, Czech Republic

2 National Defense Academy of Japan, 1-10-20 Hashirimizu, Yokosuka, Kanagawa 239-8686, Japan 


\section{Graphic abstract}

Set - up of measurement

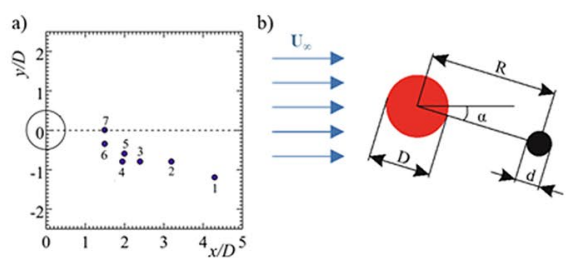

POD modes - identification of structures (two cylinders)
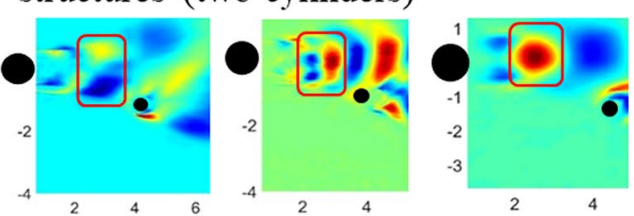

POD modes - identification of structures (single cylinder)

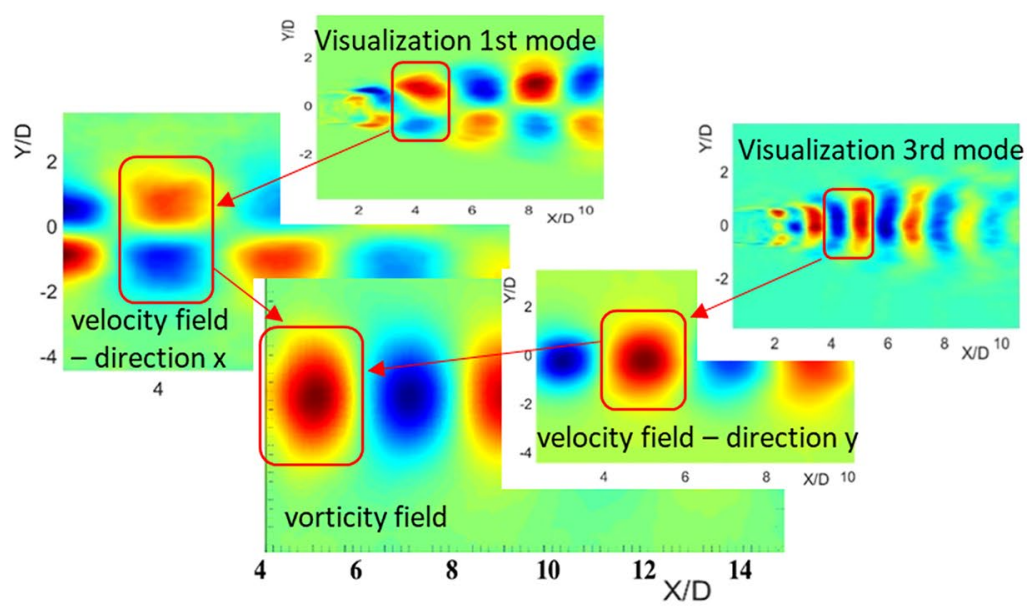

Analyzing of time dependent POD coefficient

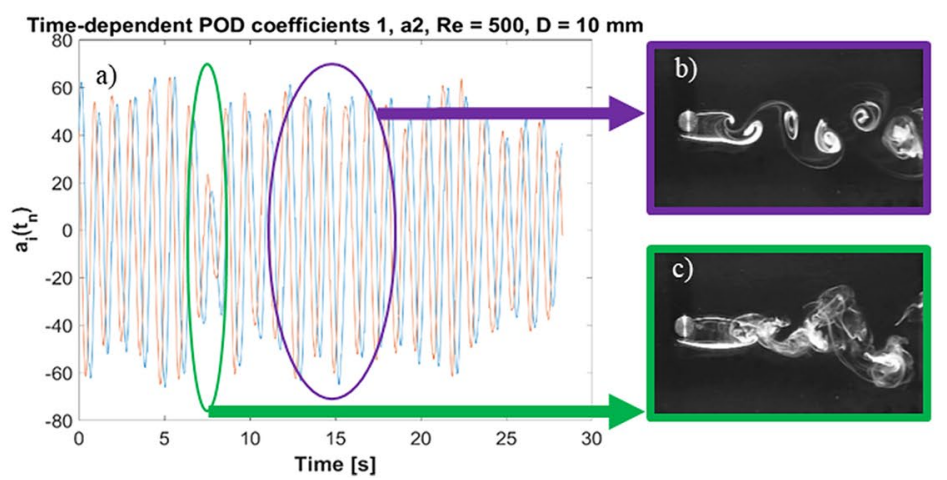

\section{Introduction}

\subsection{POD method}

Proper orthogonal decomposition (POD) is a technique for solving and analyzing the characteristics of flow fields and their structures. Its use has increased significantly in recent years. This method facilitates the analysis of large datasets by reducing the order description process. This leads to easier identification of processes that occur in the system under study. Using the POD method, physical, mathematical, biological, and environmental phenomena can be investigated and characterized owing to the fact that the dominant features and trends of processes can be recognized (Holmes et al. 1996). The POD method is known as Karhnunen-Loéve decomposition, principal component analysis, singular system analysis, and singular value decomposition. Lumley (1967) was among the first to relate the POD method to turbulent flow. The advantages of the POD method-when used to study nonlinear systems such as turbulent boundary layers-have been highlighted in Breuer and Sirovich (1991). Berkooz et al. (1993) discuss, in detail, the use of the POD method for turbulent flow. They show how POD can be used to analyze and model turbulent flow. Because this method makes it possible to simultaneously analyze and combine space-time data (Aubry 1991), other authors began to use the POD method to solve flow problems, especially in connection with the development of new experimental methods such as particle image velocimetry (PIV) (Wang et al. 2014; Graftieaux et al. 2001; Gurka et al. 2006; Hyhlik et al. 2013 and others) and computational fluid dynamics (CFD) (Frederich and Luchtenburg 2011; Le Clainche et al. 2015 and others). 
By applying the POD method to the data obtained by measuring or investigating the flow, it is possible to ascertain flow patterns that correspond to the structures with the greatest energy contribution to the flow. These structures do not necessarily correspond to coherent flow structures, but rather correspond to events that statistically contribute most to the current energy (Kostas et al. 2005). The velocity field, the vorticity field (Tang et al. 2015), the pressure field, and the flow visualization (Brevis and Garcia-Villalba 2011) can be used as input data to be processed by the POD method. By analyzing the flow field around two cylinders using the POD method, it is possible to obtain both the dominant frequencies occurring in the cylinder wakes and the structures that have the greatest influence on the formation of the wake. From the obtained results, it is possible to reconstruct the flow using a selected number of modes for a selected time period. However, Graham and Kevrekidis (1996) demonstrated that time averaging is not always the most beneficial for capturing dominant phenomena in the observed system. Another problem with using the POD method is interpreting the first mode in a turbulent flow. This is because the energy of the monitored signal is divided between the two largest modes (Kevlahan et al. 1994).

Nevertheless, the POD method simplifies the analysis of complex problems such as the flow around one or more cylinders, making it possible to ascertain other possibilities for its use. As mentioned previously, the velocity and vorticity fields are most often analyzed using this method. Nevertheless, the analysis of visualization data can also be conducted using this method. Further, there is a lack of research on this topic. However, Brevis and Garcia-Villalba (2011) demonstrate that POD can provide useful information about the flow structure and the energies of individual structures from the visualization of the flow field behind a single cylinder. Schmid et al. (2011) use the dynamical mode decomposition (DMD) method on Schlieren helium jet snapshots. However, the question arises as to how the POD method (used for PIV or visualization data) can be applied to solve flow problems around two cylinders in a staggered configuration.

When using the input data from a visualization for the POD method, it is necessary to take into account that there is only a visual change instead of a physical quantity. Therefore, it seems very useful to investigate more thoroughly how the analyzed data can be interpreted.

\subsection{Flow around two cylinders in a staggered configuration}

Solving the flow field around two cylinders is complex and requires the consideration of several variables. Flow behavior around a single-cylinder has been described by several authors, such as Roshko (1954), Williamson (1996), and Zdravkovich (1997). If there is another body close to a cylinder, such as another cylinder, the wake changes its behavior. The shear layers of both cylinders interact, along with the vortices and wakes. In his review, Zdravkovich (1977) examined the results of studies on the interactions between two cylinders in tandem and staggered configurations. In his paper (Zdravkovich 1987), he describes four areas-based on the measurement of forces-that can be found in the wake behind the cylinder. Strykowski and Sreenivasan (1990) define regions of influence of the second cylinder based on experimental measurements (CTA, LDV, and visualization) and numerical simulations for low Reynolds numbers $(R e=40-80)$ and different cylinder diameter ratios $(D / d=3-20)$. Hwang and Choi (2006) attempted to numerically find areas in the wake of the cylinder that, if affected, would reduce instability in the wake. Choi et al. (2008) compared these areas with the regions defined by Strykowski and Sreenivasan (1990). The three regions behind the cylinder are the result of comparing these researches, which have common intersections in some parts. An extensive study of visualizations and PIV measurements was performed by Sumner et al. (2000). Research has been conducted for $D / d=1$ and Reynolds numbers between 850 and 1900. The authors have defined nine characteristic types of flow around two cylinders that change the behavior of vortex structures and shear regions in the wake. $\mathrm{Hu}$ and Zhou (2008) $(R e=7000)$, Zhou et al. (2009) (Re from 1500 to $20 \times 10^{5}$ ) and Wong et al. (2014) (Re from 1500 to $20 \times 10^{5}$ ) show, in their studies, how similar configurations look at higher Reynolds numbers. They define the areas of four specific regions behind the cylinder for three Reynolds numbers.

Most of the studies presented here, which led to the discovery of regions and their characteristics, were based on experimental measurements and optical observations of the flow field of cylinders of the same diameter. However, these studies did not assess what happens in the wake when the diameter ratio of the cylinders changes, what is the main dominant structure in the flow, and how much it affects the phenomena occurring in the wake.

\section{Experimental details}

\subsection{Experimental setup}

The POD method was applied to the data obtained by researching the wake behind two cylinders in a staggered configuration (Vitkovicova and Yokoi 2019). To investigate the processes taking place in the wake of two cylinders, measurements were performed for a combination of cylinders with three diameter ratios $D / d=1,1.67$, and 2.5 , where $D$ is the diameter of a cylinder placed in front (which was $10 \mathrm{~mm}$ in all cases) and d indicates the diameter of 
Fig. 1 Setup and notation of two cylinders in a staggered configuration: a location of the center of the second cylinder behind the first cylinder, $\mathbf{b}$ dimensions (a)

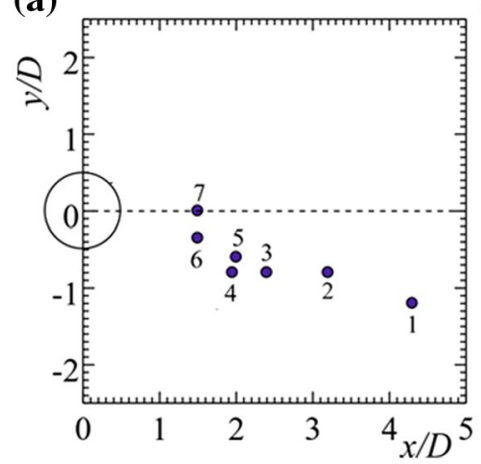

(b)

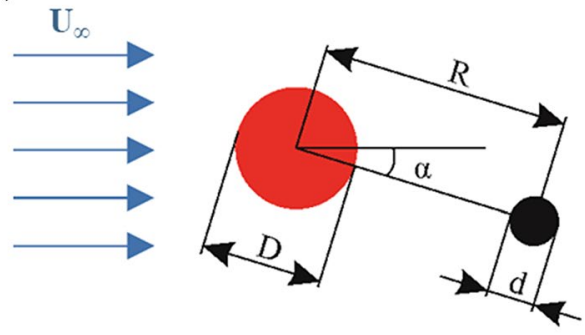

the cylinder downstream. The diameter of the rear cylinder was changed for each combination: $d=10 \mathrm{~mm}$ for $D / d=1$, $d=6 \mathrm{~mm}$ for $D / d=1.67$, and $d=4 \mathrm{~mm}$ for $D / d=2.5$. The length of all cylinders was $300 \mathrm{~mm}$. Aspect ratios $(L / D)$ had values of 75 (for $d=4 \mathrm{~mm}$ ), 50 (for $d=6 \mathrm{~mm}$ ), and 30 (for $d=10 \mathrm{~mm}$ ). The positions of the second cylinder have been chosen at seven points, as shown in Fig. 1a. Table 1 shows the coordinates of these positions and Fig. $1 \mathrm{~b}$ defines the dimensions.

The first cylinder was placed between two plates that were part of the tunnel wall. For CTA measurements, circular plates were added to the wall. A second cylinder was placed between the positioning plates. In the longitudinal direction, the spanwise uniformity of flow was checked by measuring multiple hot wire probes at the same distance from the cylinder at a given moment in time for Reynolds numbers between 600 and 8700 . In the case of a single cylinder, it was found that the values of the frequencies measured by the fluctuations were identical, given the measurement accuracy.

In all cases, Reynolds numbers $\left(R e=U_{\infty} D / \nu\right)$ are relative to the diameter $D$ of the front cylinder, the free stream velocity $U_{\infty}$, and kinematic viscosity $\nu$.

\subsection{Experimental methods and facilities}

Particle image velocimetry (PIV) measurement and visualization data are the basis for POD analysis. From this data, patterns of individual modes and their relative energy, along with the frequency of patterns of structures for each mode were identified. These frequencies were compared with those obtained from CTA measurements in a grid of points in the cylinder wakes because this method facilitates a detailed overview of the frequencies occurring in the flow field.

\subsubsection{Visualization}

Visualization measurements were performed in a small circulating water tunnel with a cross-section of $300 \times 200 \mathrm{~mm}$ and a measuring space length of $1200 \mathrm{~mm}$. The tunnel velocity was adjusted using a frequency transducer with a calibration curve for velocity dependence, where the measurement uncertainty was $\pm 1 \%$ of the current velocity. To visualize the structures and flow around the cylinders, a pouring streak method was used to visualize the flow. A different color ink was made to flow to the surface of each cylinder. Two ports on the cylinder were positioned at $\pm 60^{\circ}$ from the stagnation point of the cylinder where the ink was evenly discharged, to keep the cylinder boundary layer intact. The observed area was illuminated by a $500 \mathrm{~W}$ halogen light. The video was recorded using a video camera with a CCD chip and a recording device at a frame rate of $29 \mathrm{fps}$. The mounting of the cylinders was similar to that of the wind tunnel-the first cylinder was fixed to the tunnel walls; the second cylinder was placed between the sliding plates.

The flow field was observed using the visualization method for cylinder diameter ratios $D / d=1,1.67$, and 2.5 for seven previously defined positions and three Reynolds numbers-500, 1200, and 2500 (Yokoi and Vitkovicova 2017). The maximum of the solid blockage ratio for $D / d=1$ was $10 \%$ in position 1 , for $D / d=1.67$ was $8 \%$ in position 1 , and for $D / d=2.5$ was $7 \%$ for position 1 . The single cylinder with the diameter $D=10 \mathrm{~mm}$ blocked $5 \%$. For each recorded position and each Reynolds number, image data was obtained and subsequently processed using the POD method.

Table 1 Coordinates of cylinder positions

\begin{tabular}{lllll}
\hline Position no. & $x / D$ & $y / D$ & $R / D$ & $\alpha\left(^{\circ}\right)$ \\
\hline 1 & 4.3 & -1.25 & 4.48 & 16 \\
2 & 3.2 & -0.8 & 3.30 & 14 \\
3 & 2.4 & -0.8 & 2.53 & 18 \\
4 & 1.95 & -0.8 & 2.11 & 22 \\
5 & 2 & -0.6 & 2.09 & 17 \\
6 & 1.5 & -0.35 & 1.54 & 13 \\
7 & 1.5 & 0 & 1.50 & 0 \\
\hline
\end{tabular}


Fig. 2 Error in Strouhal numbers for position 6
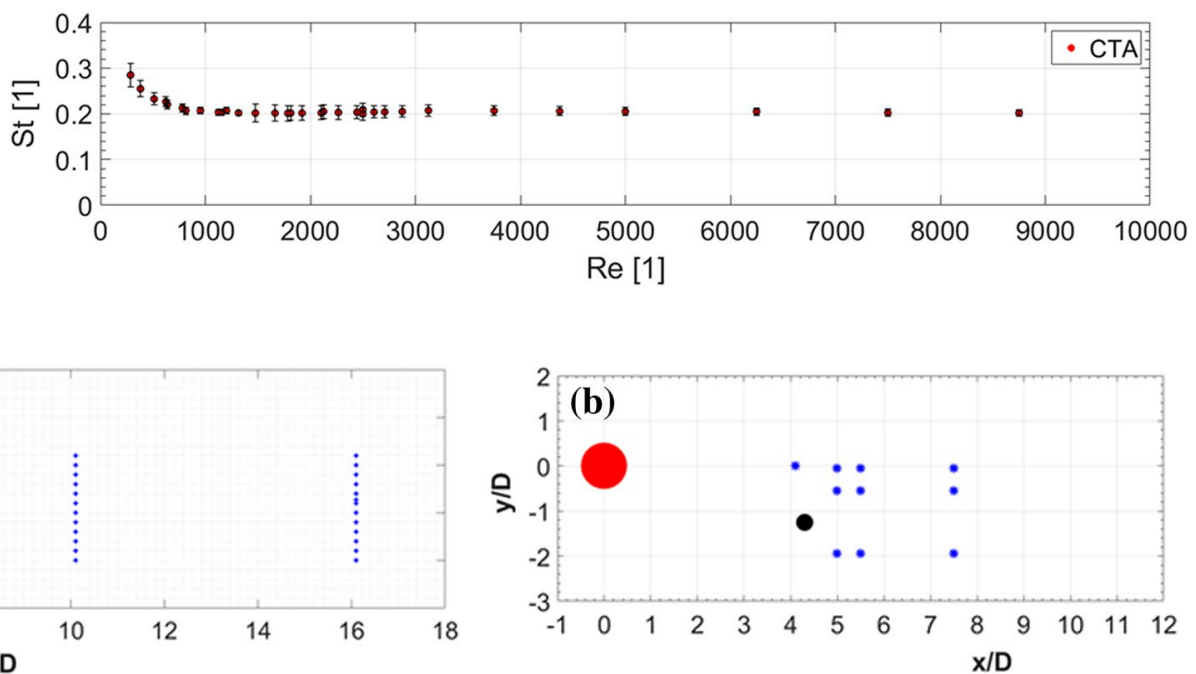

Fig. 3 Grids of measured points behind the cylinders using the CTA method: $\mathbf{a}$ in one plane, $\mathbf{b}$ in two planes

\subsubsection{CTA method}

The CTA method was chosen to accurately determine the frequencies in the wake behind the cylinders and evaluate the Strouhal numbers.

An Eiffel-type wind tunnel with a closed measuring space was used for CTA measurements. The tunnel velocity was determined using the pressure drop on a nozzle-Rosemount pressure transducer $( \pm 1 \mathrm{~Pa}$ measurement accuracy) - and verified using an OMNIPORT 20 HA040402 velocity probe for 0-20 m/s and OMNIPORT 20 HA040401 calibrated for 0-2 m/s. The uncertainty for velocity measurement was determined to be $\pm 0.04 \pm 1 \%$ of the velocity measured for the velocity range from 0.08 to $2 \mathrm{~m} / \mathrm{s}$ and $\pm 0.02 \pm 2 \%$ of the velocity measured for the velocity range of $2-8 \mathrm{~m} / \mathrm{s}$. The measurement uncertainty for the Strouhal number was dependent on the velocity. The influence of uncertainties is shown in Fig. 2, where the maximum possible error in the calculation of the Strouhal number for CTA measurements (position 6) is shown.

The turbulence intensity in this tunnel was around $1.1 \%$. The length of the measuring space was $750 \mathrm{~mm}$ and its cross-section had dimensions $300 \mathrm{~mm} \times 200 \mathrm{~mm}$. Two measurement systems were used to measure flow fluctuations. The first was a MiniCTA 54T30 system (Dantec Dynamics) with one 55P16 HW probe, and the other was a multi-channel StreamWare Pro system (Dantec Dynamics), with two 55P11 HW probes. The solid blockage ratio was the same as that in the circulating water tunnel used for visualization.

The frequencies for the two-cylinder diameter ratios$D / d=1$ and 2.5-were measured using the MiniCTA system. In these cases, the hot-wire probe was positioned in 120-140 locations in the cylinder wake in the same plane for 16 Reynolds numbers, within the range of 285-2600. The
StreamWare Pro multichannel system was used to measure the fluctuation change not only in time but also according to the position of the probe in the wake. Measurements using this system were performed for one cylinder diameter ratio, $D / d=2.5$, and for 15 Reynolds numbers in a range of about 285-2900. The probes were always placed in the plane perpendicular to the direction of flow at a distance of 41-75 mm behind the first cylinder. The spacing between the probes was $52 \mathrm{~mm}$. The sampling rate used in both measurement systems was $10 \mathrm{kHz}$. Figure 3 a shows a grid in one plane and Fig. $3 \mathrm{~b}$ shows a grid of points measured in two planes, $X Y$, behind the first cylinder.

\subsubsection{PIV measurement}

PIV measurement was performed in a circulating wind tunnel with an open test section. The turbulence intensity in this tunnel was about $1 \%$. The outlet nozzle had an octagonal shape with a maximum width of $360 \mathrm{~mm}$ and a maximum height of $200 \mathrm{~mm}$. The test section was $395 \mathrm{~mm}$ long. The tunnel velocity was determined and verified in the same manner as CTA measurements. The PIV measurement uncertainty was determined to be $5 \%$ at a displacement of 1-10 pixels for the velocity field, and the vorticity field reached an uncertainty of $10 \%$.

The measuring apparatus consisted of a $1 \mathrm{~W}$ laser diode (532-mm wavelength emission) with a lens array for the laser sheet and a high-speed Olympus i-SPEED 3 camera with a Nikkor lens with a focal length of $85 \mathrm{~mm}$, a resolution of $1280 \times 1024$, a maximum frequency of $150,000 \mathrm{fps}$, and a full resolution of the maximum frequency of $2000 \mathrm{fps}$. The Safex Fog Generator was used to create seeding particles. The cylinders were placed between two plexiglass platesthe first cylinder was firmly mounted on the stationary 
plates; the second cylinder was attached to the sliding plates. The maximum solid blockage ratio of this wind tunnel for $D / d=1$ was $9.7 \%$ in position 1 , for $D / d=1.67$ was $7.7 \%$ in position 1 , for $D / d=2.5$ was $6.8 \%$ for position 1 . The single cylinder with a diameter of $10 \mathrm{~mm}$ blocked $4.8 \%$.

The velocity fields were measured for $R e=500$ and for the cylinder ratio $D / d=2.5$.

\subsection{Proper orthogonal decomposition}

As mentioned in the introduction section, the POD method is a statistical technique that can be used to analyze events that are expected to have certain dominant recurring phenomena (Chatterjee 2000). This method can be used to determine the phenomena that contribute most to the energy of the flow, depending upon the input variable - this is the maximization of the square of the variable (Kostas et al. 2005). For example, if the variable is velocity, the structures related to kinetic energy are highlighted in the POD modes. The POD modes are, therefore, related to the events that statistically contribute the most to the energy relative to the input variable.

There are two ways to obtain dominant modes: the direct method and the snapshots method (Holmes et al. 1996). Both approaches are based on the same assumption that there is an instantaneous fluctuation component of the flow field, which can be expressed as:

$u_{N}(x, t)=\sum_{i=1}^{N} a_{i}(t) \varphi_{i}(x)$

where $u(x, t)$ represents the fluctuation component, $a_{i}(t)$ represents the time-dependent POD coefficients, and $\varphi_{i}(x)$ represents the eigenfunction of the POD modes.

The snapshot method, formulated by Sirovich (1987), makes it easier to find the solutions for coefficients and functions. Therefore, it is advantageous to select this method when analyzing a large amount of experimental data. Since this method is used to process the data presented in this work, only this approach will be discussed in the text.

Before decomposition, it is necessary to construct a matrix of fluctuating components $U=\left[u 1, u 2 \ldots u_{N}\right]$ defined in space and time in $\mathrm{M}$ points and $N$ frames. Fluctuating components can be both scalar and vector functions. For example, if the input data is a velocity field, the $U$ matrix is constructed from both velocity components:

$$
\begin{aligned}
\boldsymbol{U} & =[u(:, 1), u(:, 2, \ldots u(:, N))] \\
& =\left[\begin{array}{lll}
u(1,1) & u(1,2) & \ldots u(1, N) \\
u(2,1) & u(2,2) & \ldots u(2, N) \\
\vdots & \vdots & \vdots \\
u(M, 1) & u(M, 2) & \ldots u(M, N) \\
w(1,1) & w(1,2) & \ldots w(1, N) \\
w(2,1) & w(2,2) & \ldots w(2, N) \\
\vdots & \vdots & \vdots \\
w(M, 1) & w(M, 2) & \ldots w(M, N)
\end{array}\right] .
\end{aligned}
$$

The covariance matrix $G$ is solved in the first step to find the POD coefficients and modes for the $N$ matrix with a fluctuating flow component $U$, which is determined by:

$\boldsymbol{G}=\boldsymbol{U}^{T} \boldsymbol{U}$

The next step is to find the eigenvalues and eigenvectors of the matrix $G$ from the relation:

$\boldsymbol{G} v^{i}=\lambda^{i} v^{i}$,

where $v^{i}$ are the eigenvectors and $\lambda^{i}$ are the eigenvalues. From the eigenvalues sorted in descending order $\left(\lambda^{1}>\lambda^{2}>\lambda^{3} \cdots>\lambda^{N}\right)$, eigenvectors $v^{i}$, and the fluctuation matrix $U$, it is possible to obtain POD modes using:

$\varphi_{i}(x)=\boldsymbol{U} \cdot v_{i} \cdot \frac{1}{\sqrt{\lambda_{i}}}$.

The POD modes are ordered in descending order according to their eigenvectors; therefore, the first modes are most important in terms of the significance of the events taking place in the observed area. They contain most of the energy of the fluctuations.

Time-dependent POD coefficients, whose frequency spectrum corresponds to the frequencies of events in the monitored area, can be expressed as follows:

$a_{i}(t)=v_{i}^{T} \sqrt{\lambda_{i}}$.

How much the individual modes contribute to the overall flow pattern can be calculated from the eigenvalues of the flow. These represent the energy ${ }^{1}$ contained in the modes, whose relative values can be expressed as follows:

$E_{i}=\frac{\lambda^{i}}{\sum_{i=1}^{N} \lambda^{i}}$

\footnotetext{
${ }^{1}$ It should be noted here that, if the POD method is used to analyze data that has no relevant physical meaning, rather than as energymore precisely, a ratio of energies - it is necessary to understand the meaning of the eigenvalues as the magnitude of the given phenomenon in the flow. However, for the sake of simplicity, $\mathrm{E}_{\mathrm{i}}$ will hereinafter be referred to as energy.
} 
Fig. 4 Image processing procedure

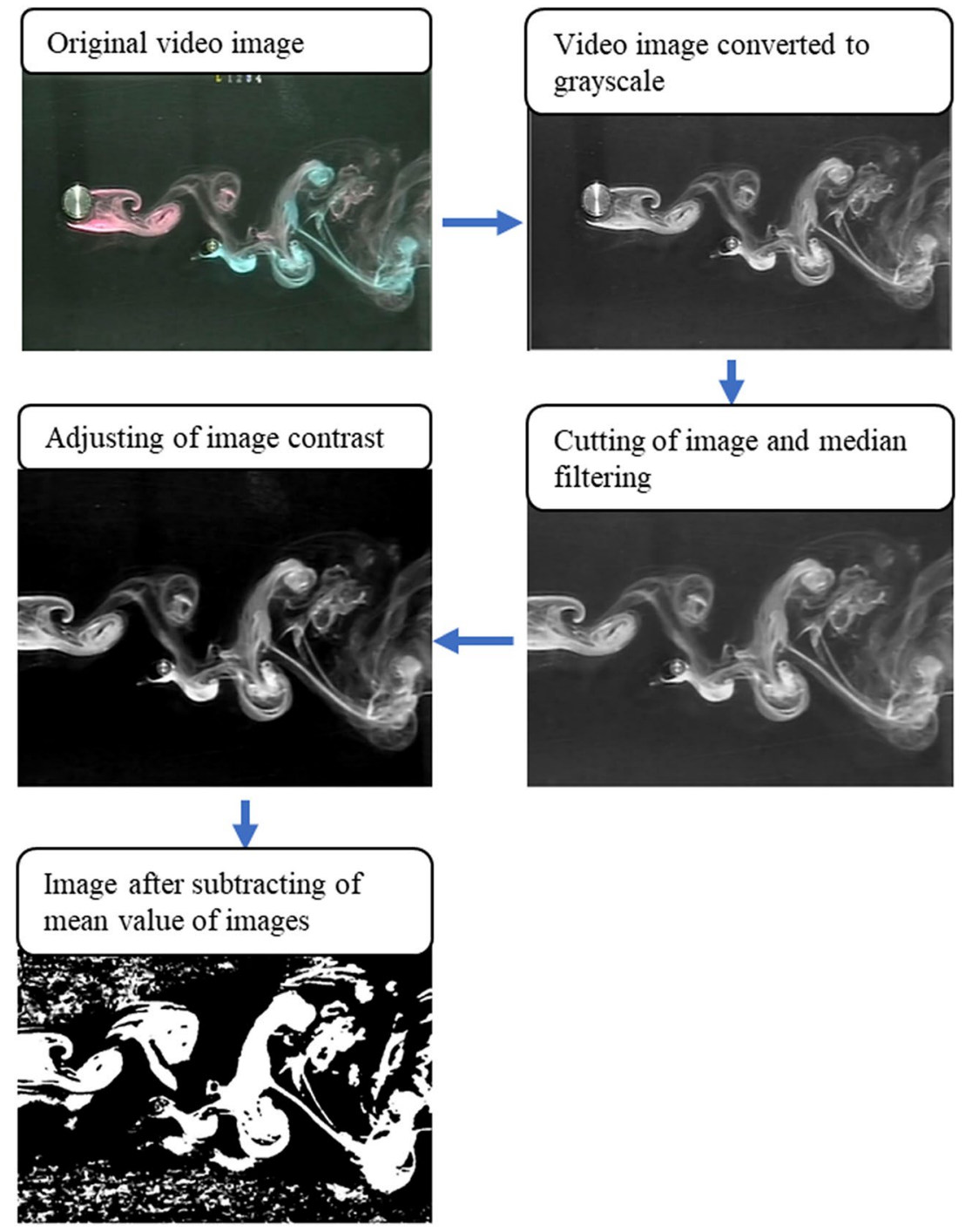

From the obtained eigenvectors and eigenvalues, it is possible to plot fields of modes, which are the dominant prevailing features of the flow. Through this, their effect on the energy of the respective phenomena can be seen. The phenomena that predominantly shape the flow pattern can be ascertained from the first POD modes. From these "first" modes, it is possible to reconstruct images of the flow at a selected time period according to the following relation:

$u\left(x, t_{n}\right)=\sum_{i=1}^{K} a_{i}\left(t_{n}\right) \varphi_{i}(x)$,

where $t_{n}$ is the selected time period and $K$ is the selected number of modes. Thus, it is possible to observe how the flow patterns contribute to the overall flow field.

\section{Methodology of experimental data processing}

\subsection{Processing of results from visualization measurements}

The video sequences visualizing the flow around the two cylinders were processed in two ways. The first was to obtain high-quality images for the description of events, from which it was possible to obtain the necessary information on the structures that are created in the wakes. This was further used to identify these structures from the POD modes. The second and main method of processing was analysis using the POD method, performed in MATLAB. The first step was to convert images to grayscale 
Fig. 5 Samples of two bad (unsaturated and oversaturated flow) and one good image
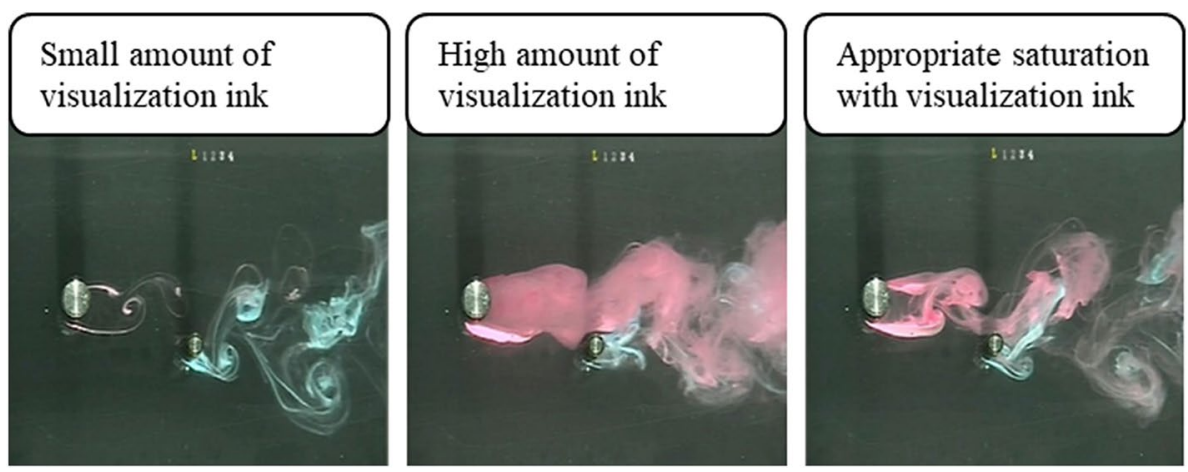

and write them into a matrix. This process had several phases, as shown in Fig. 4. Original color images from the movie were first converted to grayscale, specifically to 8-bit images, where the intensity level varied between 0 and 255 (0-black, 255-white). On the basis of these modified pictures, a section that met the criterion of constant saturation was chosen. This means that the maximum deviation in grayscale for an unaffected background is up to $10 \%$. In this case, the resulting error regarding the magnitude of the kinetic energy is up to $0.2 \%$. Smoothing with the median filter is the next phase of image editing, followed by increasing the contrast and calculating the mean of all images. In the final step, the mean value is subtracted from each frame. In this way, the dominant structures are highlighted. The noise seen at the bottom and top of the image has no effect on the mode shapes and energy. The error here is less than $0.1 \%$ and the modes are not affected. The POD modes are calculated from these adjusted images.

Subsequently, a section was selected from the video sequence that had to meet the visualization criteria. The first criterion that was important to eliminate evaluation errors was the constant saturation criterion. It is not possible to maintain the same saturation intensity and constant background shade in the circulating water tunnel over a long period of time. Therefore, only those sections that met this criterion were selected. Another important requirement was the saturation rate. If a small amount of visualization ink was present, all the movements and structures of the cylinder's wake could not be captured. In contrast, if the amount of ink was too high, owing to the saturation of the flow around the cylinders, it was also impossible to distinguish the development and changes in the structure. For this reason, sections of the same length were not always selected. Samples of bad and good images are shown in Fig. 5.

The length of the section was largely determined by the quality of the data and the requirement for capturing the complete flow period. The number of frames was usually in the range of 700-1300. How much the different number of frames had an influence on the resulting flow patterns was investigated, the influence on relative energy. Testing determined that the effect of the number of snapshots on their patterns was not apparent, and the relative energy in the first modes had a difference of up to $1 \%$ of the total energy. This difference was not necessarily due only to the number of frames, but it was an important factor if the section also recorded the complete period of the wake. After selecting the recording area, the images were adjusted using a median filter and lightened slightly. Subsequently, their mean value was subtracted from all the images such that the first mode could correspond to the structures in the wake of the cylinders and not the stream itself. The next step involved calculations of the correlation matrix, eigenvectors, and eigenvalues. The POD coefficients $a$, individual modes $\varphi$, and relative energy were obtained from the calculations. Subsequently, the courses of POD temporal coefficients for the first seven modes were plotted. The quality of the input data could be verified from them. In case the magnitude of the amplitude of coefficients $a$ was not constant and differed significantly, it was necessary to thoroughly check the selected section of the recording to ascertain whether the quality of any image was reduced in terms of the above criteria. If the given section met the requirements for all images, the dominant ten frequencies were again obtained for the first ten modes from the frequency spectrum of the POD coefficients and the Strouhal numbers were calculated. From these POD modes, the fields of eigenmodes were plotted.

\subsection{Processing of PIV measurement results}

Images were processed using the FlowManager program (Dantec Dynamics). 2500 images were evaluated for each measured position. In FlowManager, adaptive correlations were calculated for each frame $(16 \times 16$ area size with $50 \%$ overlap) and vector statistics were calculated. The vortex values were calculated from the adaptive correlations using this program. From the fluctuations of velocities at the selected points, the dominant frequencies at one point in the cylinder wake were calculated and velocity fields were drawn from vector velocity statistics in MATLAB. 
Using the POD method, the individual modes were evaluated from both velocity fields and vorticity fields in MATLAB. The procedure for conducting POD analysis was similar to that for visualization. In the case of velocity fields, relative energy was obtained from both velocity vectors, as well as the coefficient $a$ and mode $\varphi$. However, the eigenmodes were drawn in vector form from both velocity components and the eigenmodes were obtained separately for each velocity component. The dominant frequencies were obtained for each mode and the Strouhal numbers were subsequently calculated.

\subsection{Processing of CTA results}

From the CTA measurements, data (velocity and voltage fluctuations) were saved in text files and subsequently processed using MATLAB. The important findings included the dominant frequencies, calculated Strouhal numbers, and graphs of the power spectral density (PSD) for each measured point. From these courses, it was possible to determine other significant frequencies that occur in the flow. A large grid of measured points made it possible to observe the frequencies of the separate structures behind the cylinders, in detail. A comparison of the strong frequencies in the individual parts of the wake with the frequencies obtained through POD analysis contributed to the understanding of the importance of the individual structures depicted in the modes.

\section{Identification of dominant structures from POD analysis}

\subsection{Frequencies of structures}

The first measurements included the measurement of frequency and calculation of Strouhal numbers behind a single cylinder. Figure 6a shows the results of the measurements compared to the calculated values based on the relationships found in Fey et al. (1998). The value of the Strouhal number from the POD field velocity analysis is not shown here because it is identical to the value obtained from velocity field fluctuations.

Two frequencies in the wake are often reported in the case of two interacting circular cylinders in a flow field. Zdravkovich (1987) found that one frequency depends on the front cylinder and the other on the rear cylinder. Sumner et al. (2000) related the second frequency to the shear layer of the cylinders. Ishigai et al. (1972) investigated the area behind the two cylinders, where the frequencies are lower and higher. However, actual measurements do not fully correspond to these theoretical values. This is why both the strongest frequencies and other significant frequencies that appear in the frequency spectra have been closely monitored. The two values of the Strouhal number are shown in Fig. 6 for most Reynolds numbers. The second frequencies were determined from the spectral power density graphs-PSD in the case of CTA measurements - and the frequency of the 4th mode was taken as the second dominant frequency when using the POD method.

Figure 6b-h shows the dependence of $S t-R e$ for $D / d=1$, for each position. This dependence was ascertained from the CTA measurements and POD analysis from visualizations compared to the course of Strouhal numbers on a single cylinder and examples of visualization images for three Reynolds numbers-500, 1200, and 2500. At position 1 (Fig. 6b), the second cylinder evidently does not affect the resulting wake frequency significantly; the values of the Strouhal number are almost identical to those for a single cylinder. At position 2 (Fig. 6c), there are already significant changes, and the dominant frequency decreases. However, the course of the second strong frequencies in the wake, which is not as smooth as the values of the second frequencies from POD, indicates the occurrence of local wake disorders. At position 3 (Fig. 6d), a situation similar to position 2 occurs, but there are already disturbances and a change in the flow. Up to roughly the value of $R e=1000$, the values of the Strouhal number decrease, then rise up to $R e=1500$. Up to the maximum measured value of $R e=2500$, they have a constant course. Strouhal's CTA and POD numbers differ significantly in this position. This is probably due to the fact that the hot wire positioned in the grid of points in the wake is more sensitive to local changes and fluctuations that occur here, while the POD considers the observed area as a whole.

The course of Strouhal numbers at position 4 (Fig. 6e) is similar to that at position 2; however, at lower Reynolds numbers, there is a noticeable difference in that Strouhal numbers have a higher value. From a comparison of the Strouhal numbers of positions 4 and 5 (Fig. 6f) and their development with increasing Reynolds numbers, it can be assumed that the positions are identical. The flow pattern is reflected in the Strouhal numbers for the 4th mode from the POD analysis for $R e=500$ and $R e=1200$; however, at position 5 the values of the Strouhal numbers are lower. At position 6 (Fig. $6 \mathrm{~g}$ ), the Strouhal numbers follow the trend of positions 4 and 5, with the values shifted slightly lower than the values for a single cylinder. It can be seen from the smooth course of the values that there are no significant disturbances in the wake of both cylinders, which corresponds to a very good match of the Strouhal numbers from both measurements. At position 7 (Fig. 6h), the Strouhal number values are different from the assumption that two cylinders in a row will exhibit similar behavior as a single cylinder. In this case, the wake is possibly weakened by stretched shear layers, which will be reflected in the values of dominant frequencies, but only in measurements using a heated probe. 

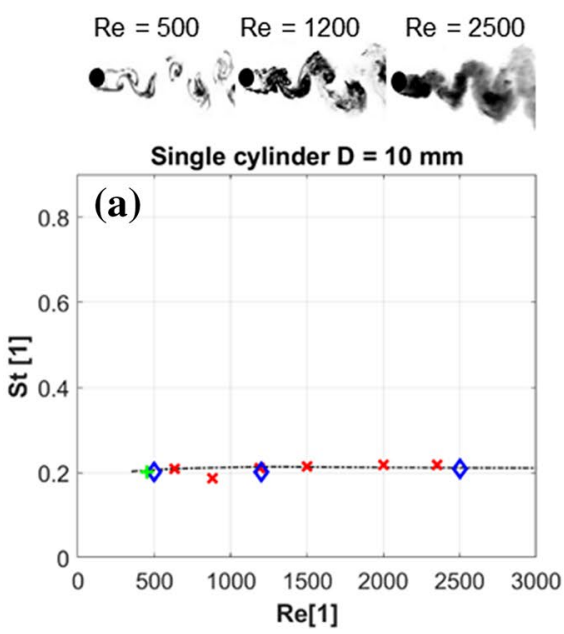

$\operatorname{Re}=500 \quad \operatorname{Re}=1200 \quad \mathrm{Re}=2500$

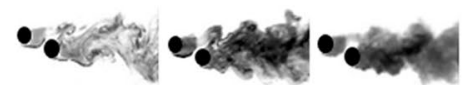

$\mathrm{R} / \mathrm{D}=2.53, \alpha=18^{\circ}$
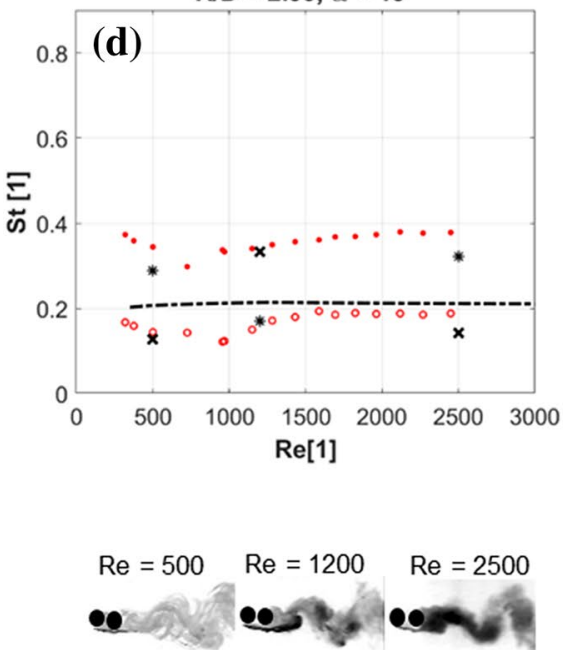

$R / D=1.54, \alpha=13^{\circ}$

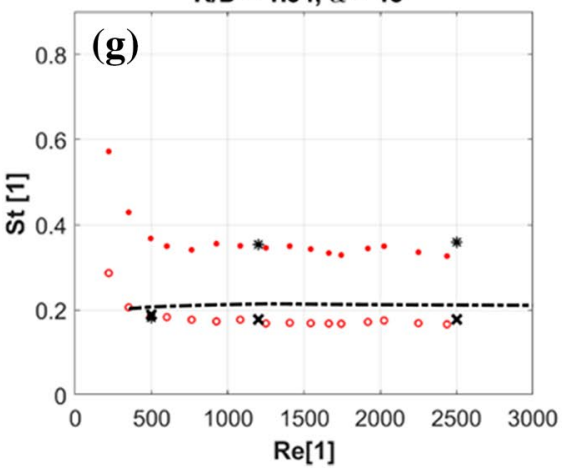

$\mathrm{Re}=500 \quad \mathrm{Re}=1200 \quad \mathrm{Re}=2500$

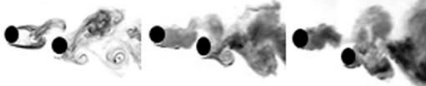

$\mathrm{R} / \mathrm{D}=4.48, \alpha=16^{\circ}$

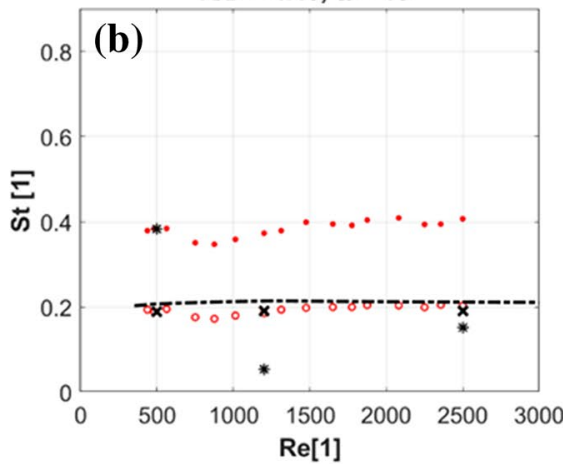

$\operatorname{Re}=500 \quad \operatorname{Re}=1200 \quad \operatorname{Re}=2500$

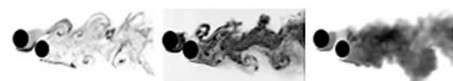

$\mathrm{R} / \mathrm{D}=2.11, \alpha=22^{\circ}$

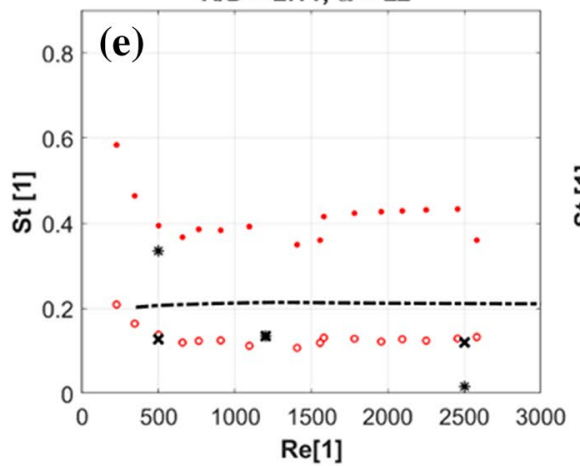

$\mathrm{Re}=500 \quad \mathrm{Re}=1200 \quad \mathrm{Re}=2500$

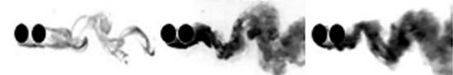

$\mathrm{R} / \mathrm{D}=1.50, \alpha=0^{\circ}$

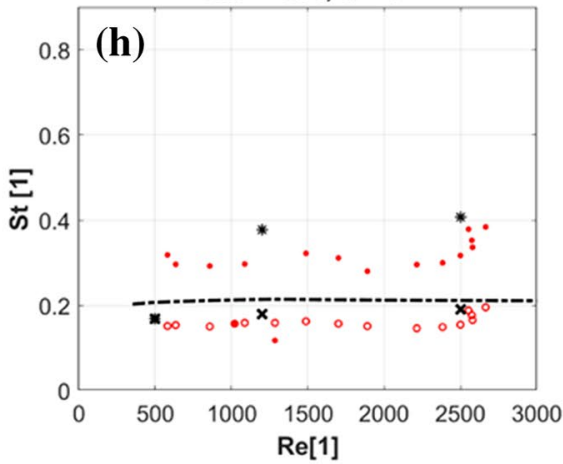

$\operatorname{Re}=500 \quad \operatorname{Re}=1200 \quad \operatorname{Re}=2500$

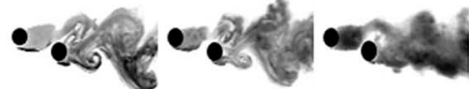

$R / D=3.30, \alpha=14^{\circ}$

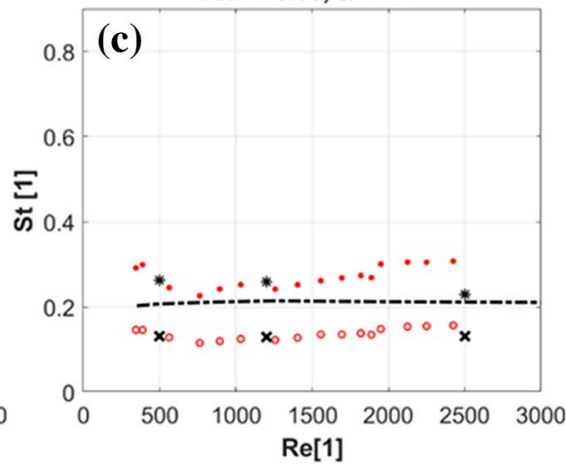

$\mathrm{Re}=500 \quad \mathrm{Re}=1200 \quad \mathrm{Re}=2500$

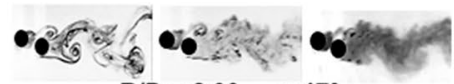

$\mathrm{R} / \mathrm{D}=2.09, \alpha=17^{\circ}$

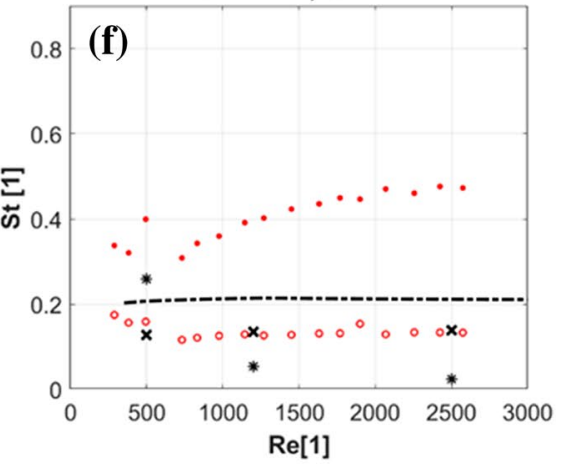

Fig. 6 Dependence of $S t-R e$ from CTA and POD analysis for $D / d=1$ and visualization of positions for $R e=500$, 1200 , and 2500 (from left to right), a single cylinder, b position 1 , c position 2 , d position 3 , e position 4 , f position 5 , $\mathbf{g}$ position 6 , and $\mathbf{h}$ position 7 

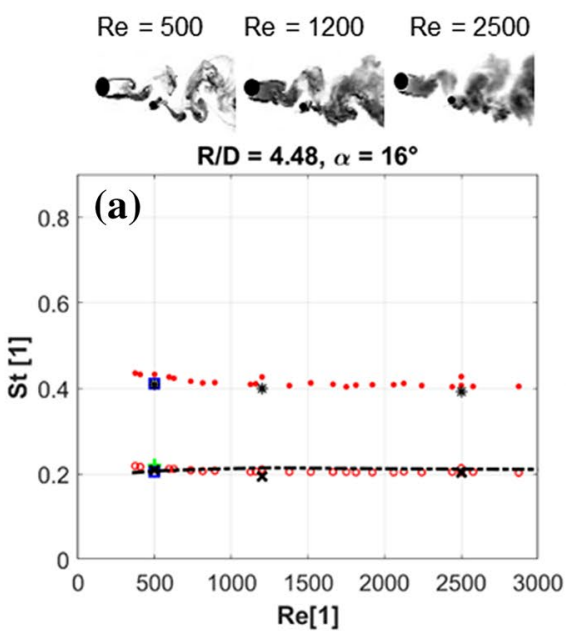

$\mathrm{Re}=500 \quad \mathrm{Re}=1200 \quad \mathrm{Re}=2500$

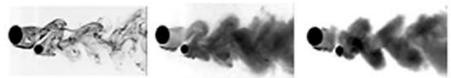

$R / D=2.11, \alpha=22^{\circ}$

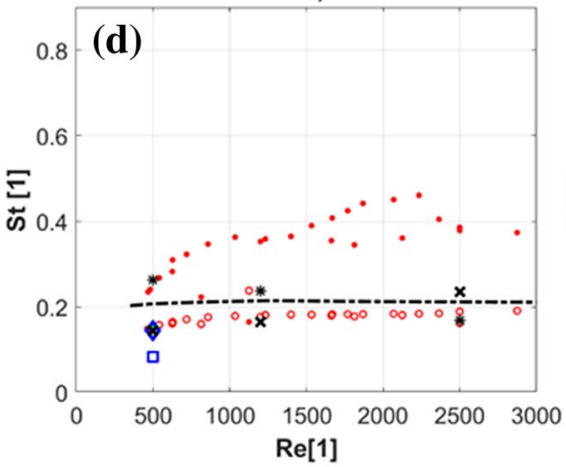

$\mathrm{Re}=500 \quad \mathrm{Re}=1200 \quad \mathrm{Re}=2500$
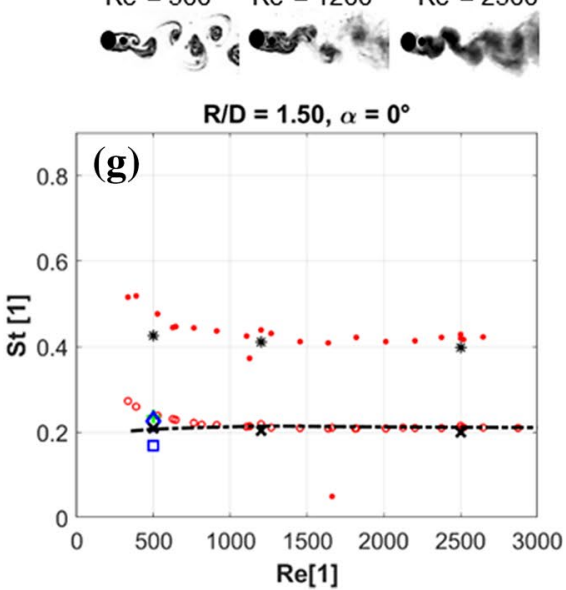

$\mathrm{Re}=500 \quad \mathrm{Re}=1200 \quad \mathrm{Re}=2500$

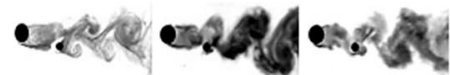

R/D $=3.30, \alpha=14^{\circ}$

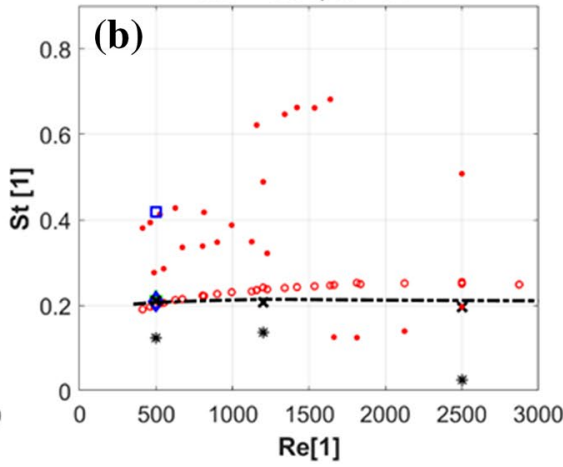

$\operatorname{Re}=500 \quad \operatorname{Re}=1200 \quad \operatorname{Re}=2500$

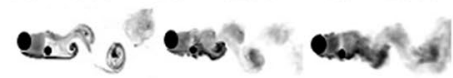

$\mathrm{R} / \mathrm{D}=2.09, \alpha=17^{\circ}$

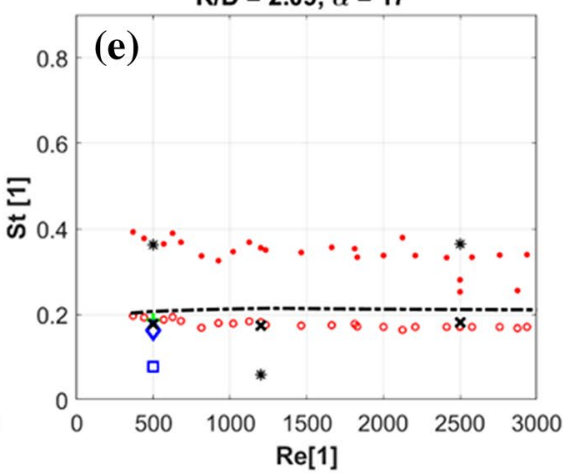

$\mathrm{Re}=500 \quad \mathrm{Re}=1200 \quad \mathrm{Re}=2500$

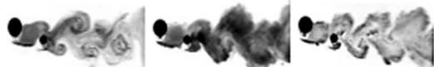

$\mathrm{R} / \mathrm{D}=2.53, \alpha=18^{\circ}$

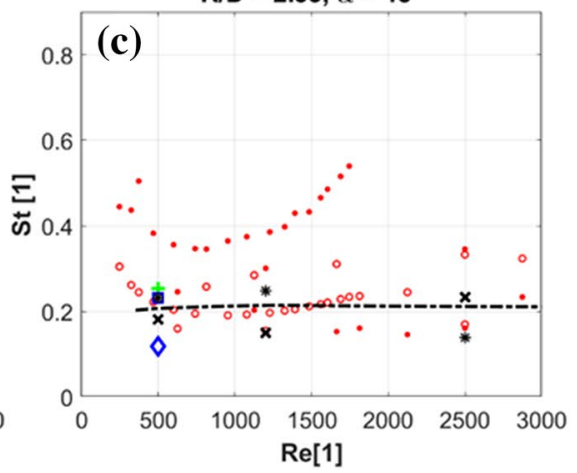

$\operatorname{Re}=500 \quad \operatorname{Re}=1200 \quad \mathrm{Re}=2500$

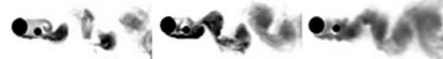

$R / D=1.54, \alpha=13^{\circ}$

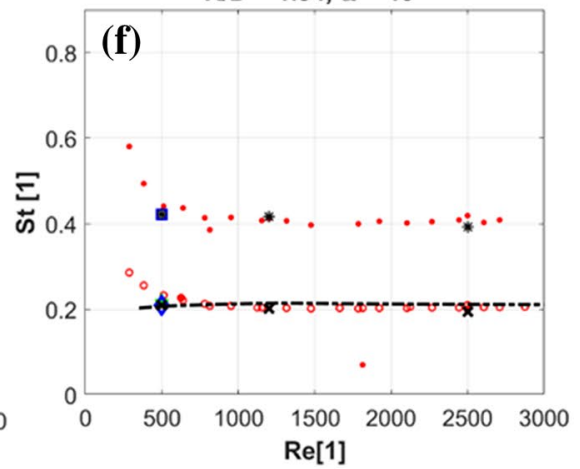

Fig. 7 Dependence of $S t-R e$ from CTA and POD analysis for $D / d=2.5$, and visualization of positions for $R e=500$, 1200 , and 2500 (from left to right), a position 1 , b position 2 , c position 3 , d position 4 , e position 5 , $\mathbf{f}$ position 6 , and $\mathbf{g}$ position 7 
Figure 7 shows the results from frequency measurements by CTA, PIV, and POD analysis for $D / d=2.5$ with single cylinder values. Further, it shows visualization images for three Reynolds numbers-500, 1200, and 2500. The Strouhal numbers at position 1 (Fig. 7a) have a similar pattern to those for $D / d=1$. At position 2 (Fig. $7 \mathrm{~b}$ ), there are already distinct differences. From the CTA measurements, it can be seen that the values of the Strouhal numbers differ slightly from the POD measurements. The reason for this is probably the same as that for $D / d=1$ at position 3 . However, the Strouhal numbers of the dominant frequencies from CTA are higher than those for a single cylinder and their course shows that local instabilities occur at this position. At position 3 (Fig. 7c), the first cylinder's wake disruption is most noticeable. From the CTA measurements, it is very difficult to determine which frequency is dominant in this case, because (similar to position 2) it strongly depends on the position of the heated probe. The main reason for these frequency differences across the wake is the variation in the frequency of formation of the shear layers from the individual cylinders and the repeated disruption of the formation of the vortex street of the first cylinder by the shear layers of the second cylinder. Position 4 (Fig. 7d) does not show such a chaotic near wake. The near wake is more compressed; therefore, the vortex streets are formed behind the second cylinder. It is also clear from the graph in Fig. $7 d$ that this second cylinder position reduces the dominant frequency in the wake significantly (especially for lower Reynolds numbers). At Position 5 (Fig. 7e), no separate vortex street is formed behind the second cylinder. Compressing the near wake of the front cylinder and splitting its shear layers weakens the wake, which results in lower dominant frequencies and thus lower Strouhal numbers in comparison to a single cylinder. The rear cylinder, located at position 6 (Fig. 7f), does not significantly disturb the wake of the first cylinder, but simply introduces several possible disorders into the system. The situation here is similar to that for $D / d=1$. At position 7 (Fig. $7 \mathrm{~g}$ ), the smaller cylinder tends to shed the shear layers at a different frequency than the first cylinder. Although these shed layers are absorbed by the shear layers of the first cylinder, they cause flow disturbances. The absence of the second significant frequencies at the higher Reynolds numbers indicates a complete synchronization of the wakes and the "unification" of the two cylinders into a single body, in terms of flow.

\subsection{Structures in flow}

As mentioned previously, the main characteristics of flow are included in the first few POD modes, which result from the relative energy values of the phenomena. Usually, the shapes of the POD modes are paired, which means that the plotted patterns have similar relative energy values and very similar structures, which differ only by phase shift. Therefore, the pattern of the first and second modes will be almost identical, ${ }^{2}$ as will the third and fourth, and so on. Nevertheless, it can be seen from the analysis of a large amount of data that there are cases in which one of the first modes does not contain clear wake structures, but rather the pattern of the flow, even if the mean value is subtracted. The possible cause of this phenomenon will be discussed later.

For POD modes obtained from both velocity and vorticity field PIV data and visualization data, differently shaped structures are obtained. As mentioned in 2.3, the choice of input data affects the resulting mode patterns, as the POD analysis processes and highlights how the input quantity contributes to the overall characteristics of the flow (Kostas et al. 2005). For this reason, differences are evident, although not extensive. If the aim is to obtain information regarding the shapes of coherent structures in terms of their definition—such as in a study by Hussain (1986) and the physical approach-vorticity seems to be the most appropriate input for describing coherent structures, since vorticity is invariant according to the classical principle relativity (Kostas et al. 2005). For determining the kinetic energy's relationship with individual processes and flow patterns, the velocity field is more advantageous as input data. Given that the change in intensity of gray shade has no physical meaning, in individual modes, it is more about highlighting flow structures and drawing analogies with other physical quantities. Therefore, to understand the meaning of the eigenmodes, it is necessary to examine the obtained modes in more detail. Only in this way can a parallel be drawn between these patterns, which—in fact-describes the same processes in the flow, only each from a slightly different point of view.

\subsubsection{Single cylinder}

From the data obtained from PIV measurements, the velocity field was only analyzed for $D=10 \mathrm{~mm}$ and a Reynolds number of 450 . Figure 8 a shows the first mode of the POD from the velocity field.

Tang et al. (2015) presented similar results (input data also obtained using PIV) as those in Fig. 9. The first modes from the velocity fields (Fig. 9a) and the vorticity fields (Fig. 9b) are shown in this figure.

Figure 10 shows the first and third POD modes from the visualizations for $D=10 \mathrm{~mm}$ and $R e=500$ (the

\footnotetext{
2 This is true when the mean value of all frames is subtracted from the data. In case it is not subtracted, the first mode contains the energy of the flow.
} 

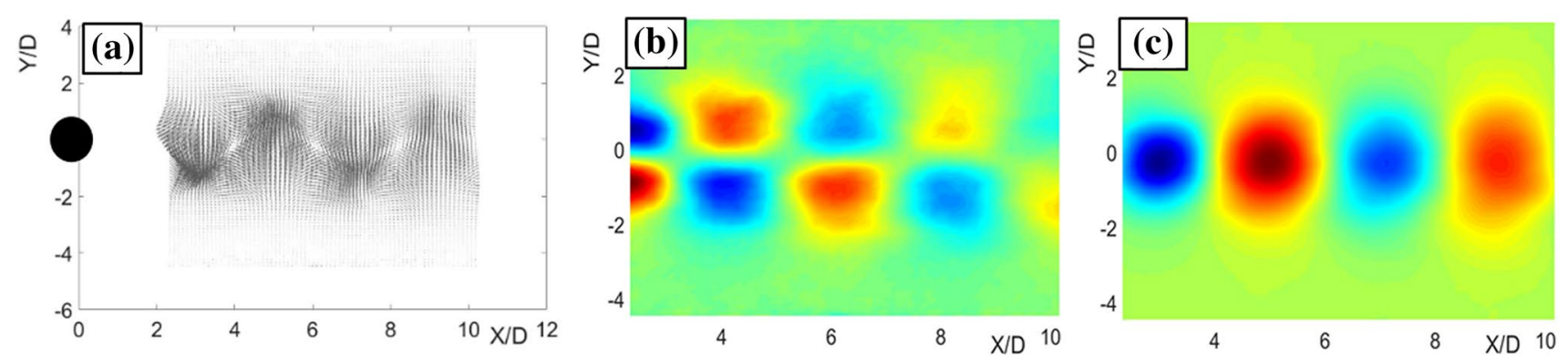

Fig. 8 First mode of the POD from the velocity field, $D=10 \mathrm{~mm}, R e=500$; a vector field from both components, $\mathbf{b}$ shapes of the $x$-component, and $\mathbf{c}$ shapes of $y$-component
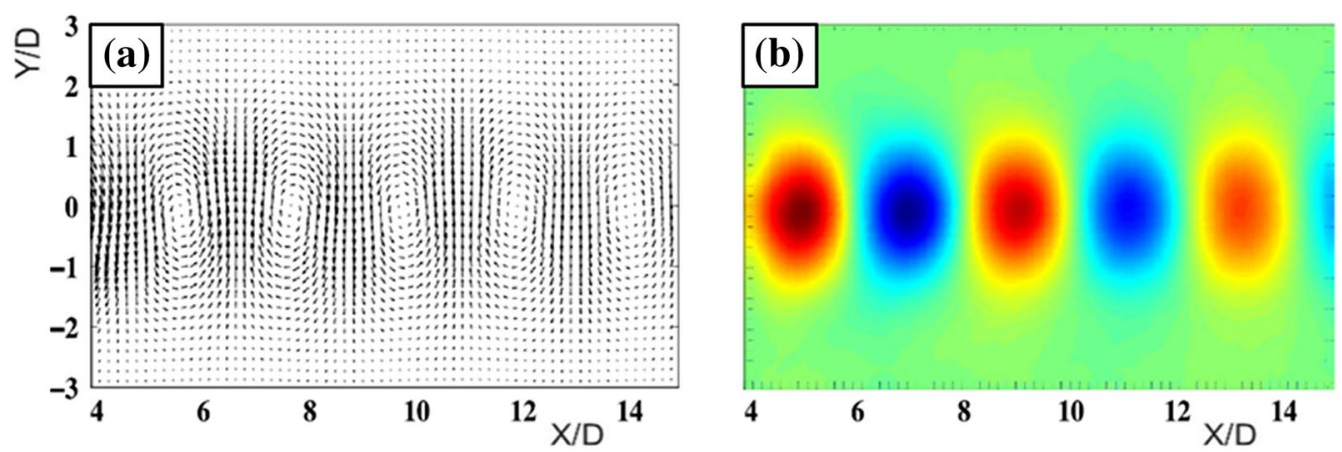

Fig. 9 First POD mode of single cylinder, $D=10 \mathrm{~mm}, R e=5800$; a velocity field, and b vorticity field, taken from Tang et al. (2015)

Fig. 10 The first and third POD modes from the visualization for a single cylinder $D=10 \mathrm{~mm}$; a mode $1, \operatorname{Re}=500$, and $\mathbf{b}$ mode 3, $R e=500$
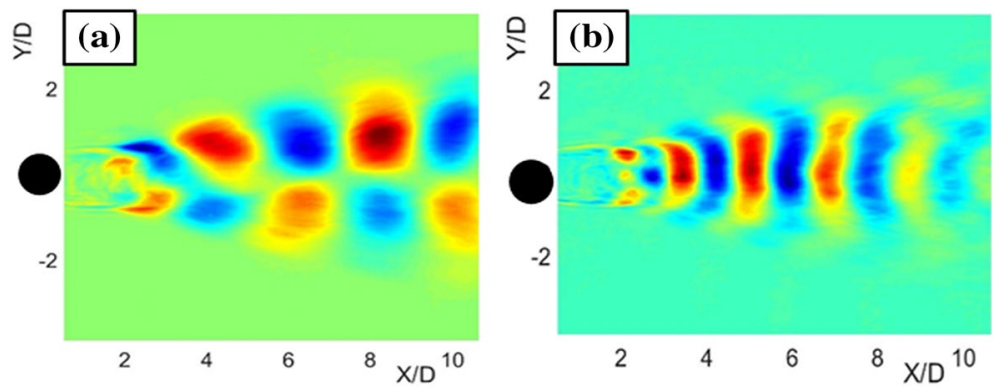

visualizations were also observed for $R e=1200$ and 2500, but since the shapes are essentially identical, they are not shown here).

Thus, in Figs. 8, 9, 10, the same Karman's vortex street is depicted, only the Reynolds numbers differ. The development and direction characteristics of the dominant structure associated with the velocity field (Figs. 8, 9a) are evident from the decomposition of the velocity fields; the first mode of the POD from the vorticity field (Fig. 9b) shows the shape of the dominant coherent structures. Figure 10 shows Karman's vortex street behind a single cylinder for one Reynolds number for the POD modes from the visualization. In the first mode, the same structure is visible for all modes (the same is true for the second mode, which is not shown here), which is anti-symmetrical to the $x$-axis and represents the translational movement of structures in the flow (Brevis and Garcia-Villalba 2011). These structures, which represent the time POD coefficient $a$, have a dominant frequency and a Strouhal number that corresponds to the Strouhal number obtained from the CTA measurements. Modes 3 and 4 (not shown here, but its characteristics are the same as those for mode 3) correspond to the transverse movement of structures (Brevis and Garcia-Villalba 2011). In this case, the structures are symmetrical about the $x$-axis and their frequency corresponds to the second strongest frequency of the CTA. The mode shapes for the individual components of the POD velocity field correspond to this statement, as shown in Fig. 8b, c. The shapes of the flow of the first mode 
Fig. 11 Identification of structures behind one cylinder from different POD data (vorticity field from Tang et al. (2015))

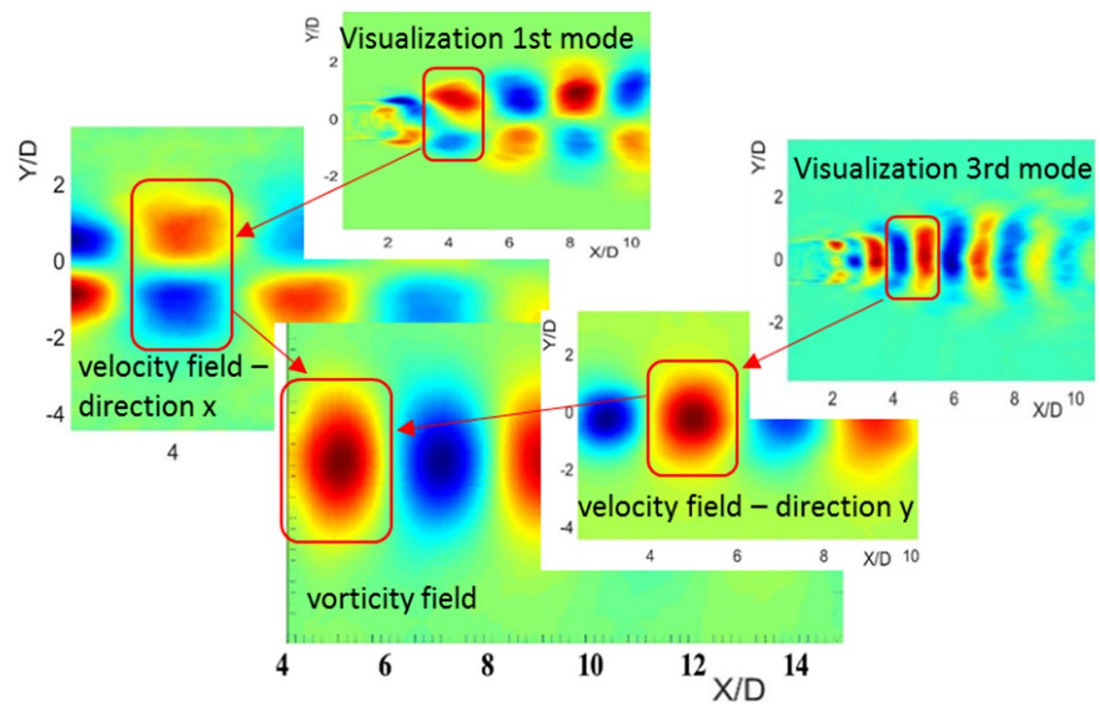

in the flow direction have the same anti-symmetrical characteristics as the first mode of the visualization. In all cases, the POD shapes in the transverse direction are symmetrical about the $\mathrm{X}$-axis; however, the shapes from the velocity and vorticity fields are similar (Fig. 9b). It is by combining the information from these results that one can understand what the flow shapes of POD modes from visualizations represent in this case.

Figure 11 shows the correlation between structures of all types of input data. The same structure is identified in the red box presented from a different perspective, from the point of view of input quantity. The first POD mode from the visualization corresponds to the first mode from the velocity field in the $x$-direction, both in pattern and in Strouhal number. Both show movement of the structures in the flow direction. The third POD mode from the visualization and the first mode from the velocity field in the $y$-direction exhibit dominant motion in the transverse direction; however, there are slightly different eigenmodes and Strouhal numbers. In the case of the first mode of the velocity field in both the $x$ and $y$ directions, the dominant frequencies (and Strouhal numbers) are the same. This is not the case in the first and third modes of visualization. The third mode has twice the dominant frequency of the first mode. Further, when the eigenmodes are compared, one shape of the structure in the first mode of the velocity field corresponds to two shapes of the third mode, as shown in the figure. This explains why the dominant frequency of the third mode is twice that of the first mode. The fact that the two structures of this mode are similar to those in the first mode of the velocity field in the $y$-direction demonstrates mainly the distance from the cylinder and the dimensions of the formation.

Proper orthogonal decomposition (POD) analyses differ most in relative energy. With respect to the analysis of velocity field data, the first two modes contain $48 \%$ of the kinetic energy. Tang et al. (2015) $(R e=5800)$ reported this value to be approximately $69 \%$ and Zhang et al. (2014) $(R e=8000)$ reported $56.6 \%$. The difference could be owing to different Reynolds numbers and changes in the flow structure. Tang et al. (2015) report that the value of energy, especially the enstrophy from vorticity fields for the first two modes, is approximately $68 \%$, which is almost identical to the kinetic energy. A similar energy value indicates that, in this flow, most of the energy is concentrated in coherent structures. The energy of the structures from the POD analysis for the first two modes at $R e=500$ is about $27 \%$; for $R e=1200$, it is $37 \%$; and for $R e=2500$, it is $41 \%$. Thus, the relative energy of the first modes (in this case, for a given Reynolds number range) tends to increase with increasing Reynolds numbers. This is also confirmed by the work of Brevis and GarciaVillalba (2011) $(R e=16,200)$, who report a relative energy value for the first two modes of 53\% (input data from visualization). However, as shown by Zhang et al. (2014), at a certain Reynolds number, the relative energy in the first modes start to decrease, at least in the case of data from velocity fields. Nevertheless, the question arises as to whether this applies to POD from any input data or whether it is related to a certain type of data. Further research can help explain this better.

\subsubsection{Two cylinders in a staggered configuration}

Position 1 of the second cylinder is a position that does not have such a significant effect on the entire wake. Figure 12a shows the first POD mode for $D / d=2.5$ and $R e=500$. The patterns for all $D / d$ ratios have similar characteristics; they differ mostly only in the energy of individual structures. In the POD modes obtained from the PIV data (Fig. 12b-d) from the velocity and vortex fields), shape-different structures are seen at first glance in comparison to the POD mode 

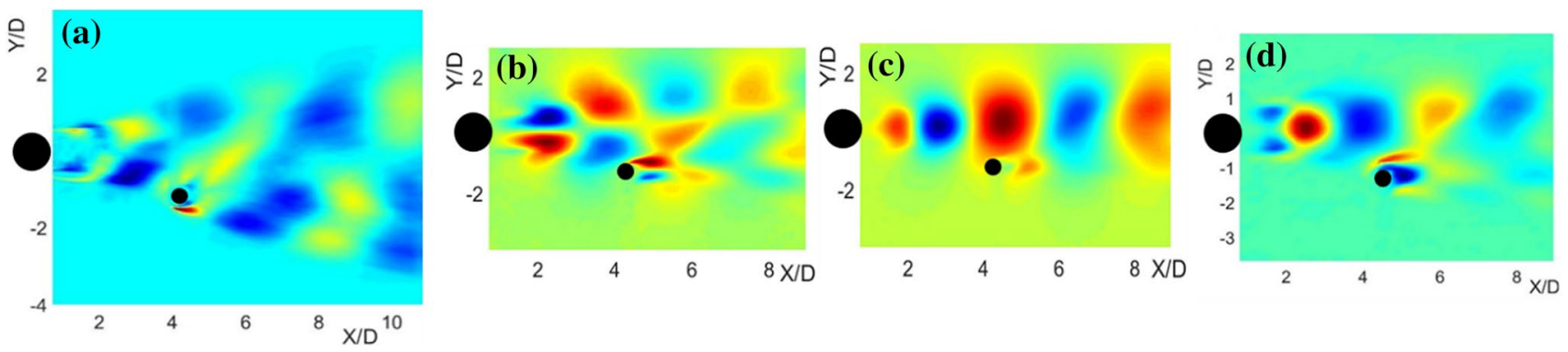

Fig. 12 First POD mode of position $1, D / d=2.5, R e=500$; a visualization, $\mathbf{b}$ velocity field in direction $x$, $\mathbf{c}$ velocity field in direction $y$, and $\mathbf{d}$ vorticity

structures from the visualization, as was in the case of the single cylinder. In the picture of the first mode of vorticity (Fig. 12d), there are four formations between the first and second cylinders. In view of what has been stated in the previous paragraphs on the patterns of the POD modes from vorticity, it can be stated that these formations are coherent structures. Two blue $\mathrm{e}^{3}$ formations indicate two vortexes in the near wake. The following two structures are vortexes in the Karman street. The same can be seen in the first mode from the velocity fields for both the $x$ and $y$ directions. A similarity to these structures can also be found in the POD modes from visualizations, as shown in Fig. 13, where the structure (vortices) occurs for the $D / d=2.5, R e=500$, in both the first and third modes. An identical vortex is marked in the red rectangle (Fig. 13a) is mode 1, Fig. 13b is mode 3, and Fig. $13 \mathrm{c}$ is the corresponding structure for POD vorticity).

At position 2, for $D / d=1$ (Fig. 14a, b), the largest structures are formed by the outer shear layer of the second cylinder. With increasing diameter ratios of cylinders and Reynolds numbers, it is clear that the importance of the shear layers of the first cylinder increases. This can be seen from the distinctive structures below the horizontal axis of the first cylinder in Fig. 14a.

At position 3, the visualizations showed that, in the case of $D / d=1$, an unsynchronized formation of vortices from the shear layers occurs. This is evident in the first POD mode of this ratio. With $R e=500$ (Fig. 14c), no coherent structure is recognizable-the visible structure represents the flow. At higher Reynolds numbers, shear layers and their development into vortex streets appear in the first mode itself. As the $D / d$ ratio increases, the importance of the second cylinder and its vortex path increases $(D / d=1.67, R e=2500$, Fig. 14d). For position 3, for higher $D / d$ in most cases, the

\footnotetext{
3 The blue scale of the POD modes indicates negative values and the red scale indicates positive value of the eigenfunction. The color scale is not given here owing to its lack of physical meaning (in the case of POD from visualization). The color scale is only significant in terms of comparing the structures and their shapes for each mode.
}

smallest or second smallest relative energy of the structures is contained in the first two modes, and the next two modes are only slightly less. This makes it evident that the formation of clear structures in this position is disrupted.

At position 4, the wake of the front cylinder is significantly compressed and the shear layers of the first cylinder, along with the inner shear layer of the second cylinder, create vortices and synchronize with the vortex street from the outer shear layer of the second cylinder (Fig. 14e). As the Reynolds number increases, the vortex street gains greater importance for the rear cylinder and its outer shear layer, which may have a large impact on the reduction of the dominant frequency for Reynolds numbers ranging between 500 and 4000 (Fig. 14f).

At position 5, there is no significant disruption of structures, as in the previous positions. This is evidenced by the actual flow patterns in the third mode at $R e=500$, for all three $D / d$ ratios (Fig. 14g) shows the third mode for $D / d=2.5, R e=500$ ). As is the case for a single cylinder, a coherent structure (vortex) can be recognized and precisely identified, as can be found in the first vortex mode image (Fig. 14h). Overall, this position no longer has its own vortex street for the second cylinder, but the second cylinder significantly affects the overall wake and its position contributes to a significant reduction in the Strouhal number. The relative energies in the first two modes of this position are among the highest. The exception is $D / d=1$ and $R e=500$ and 1200 . A disturbance of stability in this position by the (a)

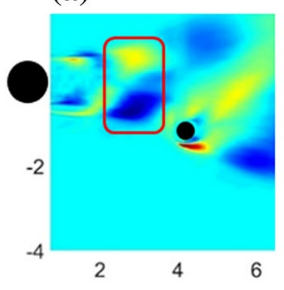

(b)

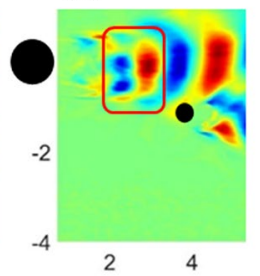

(c)

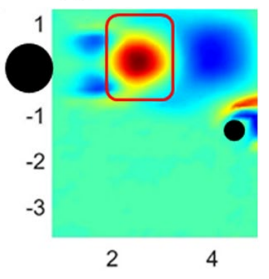

Fig. 13 Structure identification behind the first cylinder for $D / d=2.5$, $R e=500$; $\mathbf{a}$ first mode of visualization, $\mathbf{b}$ third mode of visualization, and $\mathbf{c}$ first mode of vorticity 

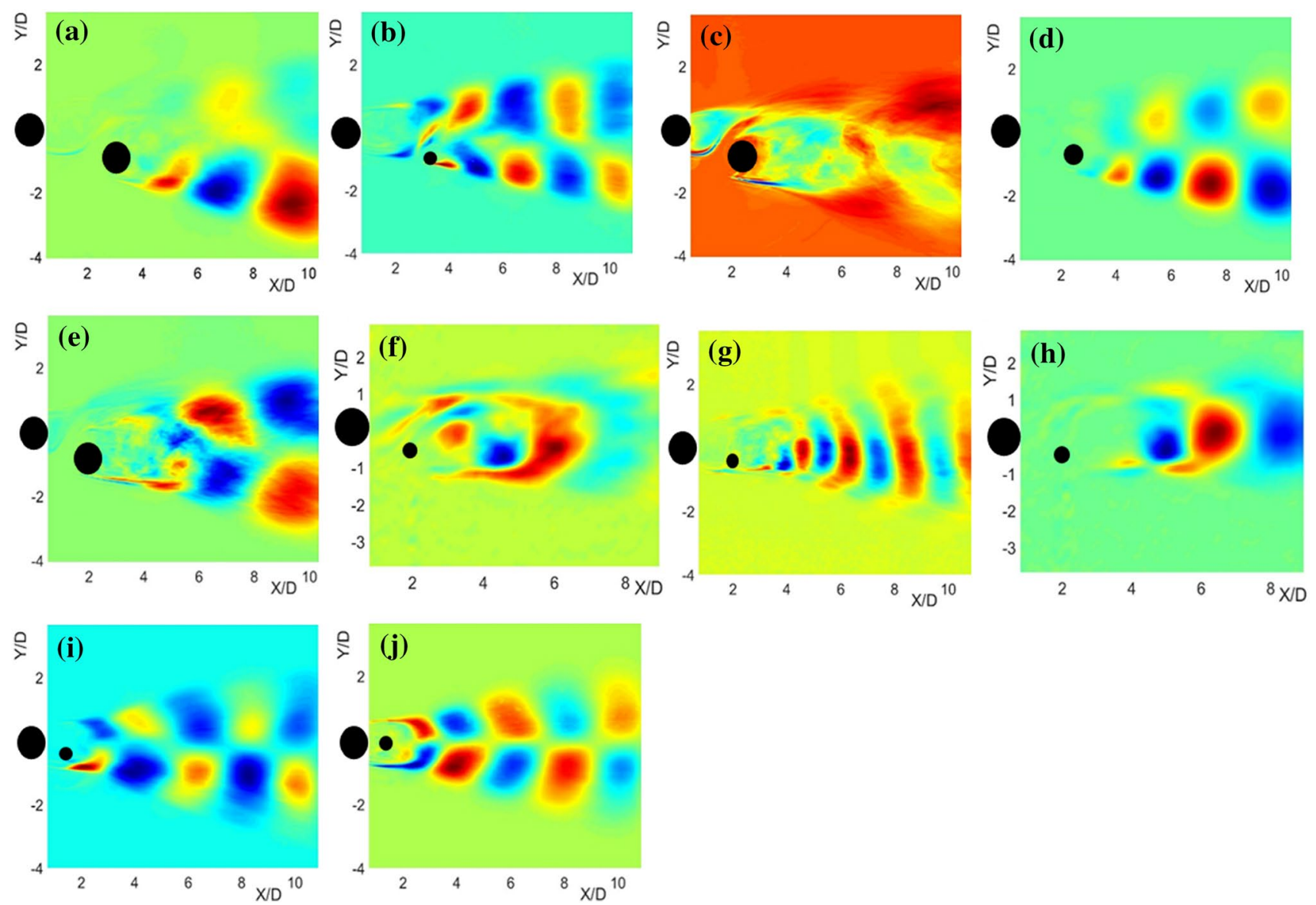

Fig. 14 POD modes, a 1st visualization mode, position $2, R e=500$, $D / d=1$, b 1 st visualization mode, position $2, R e=500, D / d=2.5$, c 1 st visualization mode, position $3, D / d=1, R e=500$, d 1 st visualization mode, position $3, \mathrm{D} / \mathrm{d}=1.67, R e=2500$, e 1 st visualization mode, position $4, D / d=1, R e=500$, f 1 st mode of vorticity, position

overcompressed near wake of the first cylinder may be the reason for this.

At position 6, the POD analysis facilitates a more clear understanding of what the source of the aforementioned disturbances could be. A significant outer shear layer in the first mode from the second cylinder is clearly visible in almost all configurations and Reynolds numbers, which is shown in Fig. 14i. With an increasing $D / d$ ratio and a slightly increasing Reynolds number, its significance reduces a small amount.

At position 7, there is already a tandem configuration, where the shear layers of the second cylinder are connected to the shear layers of the first cylinder. The POD patterns of all configurations - in both the first and third modes - are very similar to the intrinsic POD patterns of a single cylinder (Fig. 14j). Differences can only be observed at the start of the formation of dominant structures.

If the influence of measurement uncertainties on the conclusions drawn from the data analysis conducted would be

4, $D / d=2.5, R e=500, \mathbf{g} 3 \mathrm{rd}$ visualization mode, position $5, D / d=2.5$, $R e=500, \mathbf{h} 1$ st mode of vorticity, position $5, D / d=2.5, R e=500, \mathbf{i} 1 \mathrm{st}$ visualization mode, position $6, D / d=2.5, R e=500$, j 1 st visualization mode, position $7, D / d=2.5, \operatorname{Re}=1200$

studied, it could be said that the impact is very small. This is owing to the fact that the whole complex of information about the behavior of structures has been studied from a large volume of different types of data, such as mechanisms and phenomena in the flow field around two cylinders, the velocity field, vorticity field, shapes of structures, and Strouhal numbers.

\subsubsection{Stability losses in the wake}

However, as mentioned before, when analyzing a single cylinder using POD, flow patterns occurred in the first seven modes, in which there were no structures corresponding to vortices. However, in these modes, the shape of the flow was recognizable. This is despite the fact that the mean flow value was subtracted from images or fields of vorticity and velocity prior to POD analysis. These modes, with flow patterns, occur in all three types of processed data. This phenomenon has also been reported by some other authors, 
Fig. 15 Loss of stability in the vortex street, a coefficient and time dependence for a single cylinder, $D=10 \mathrm{~mm}$, $R e=500$ - an example of an area with a stable vortex path is indicated in violet, an example of an area with a loss of stability is indicated in green, $\mathbf{b}$ stable vortex street, and $\mathbf{c}$ loss of stability in the vortex street

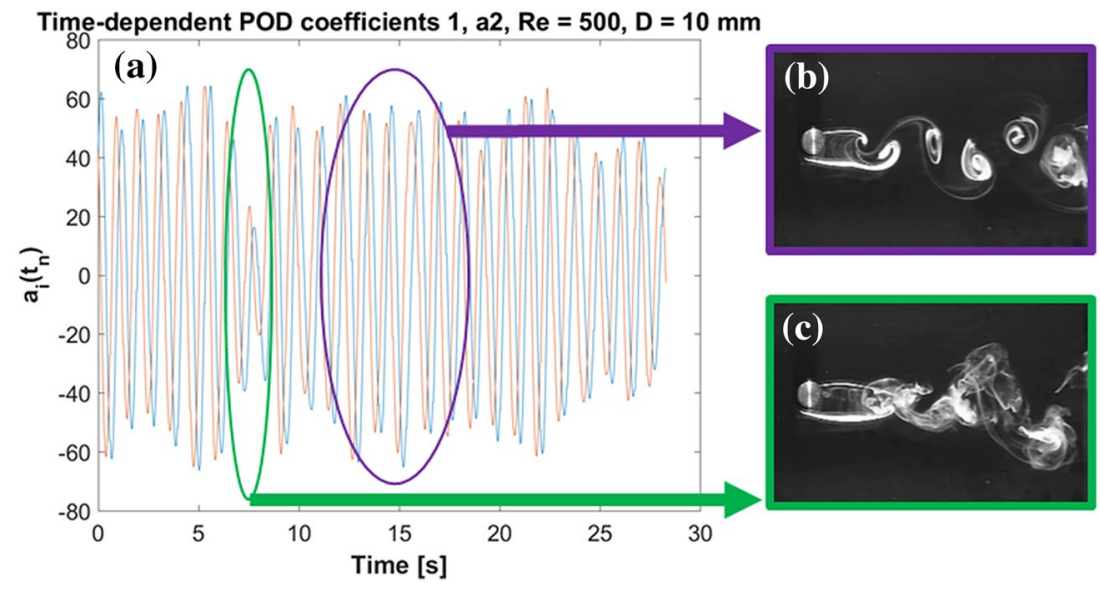

such as Zhang et al. (2014). The reason for this was not fully elucidated in this work; however, videos from the visualizations suggested where this could be the cause. Almost every configuration setup and even single cylinders have seen very short stability losses, at which the vortex street disappeared in the wake. In Fig. 15, this anomaly is shown. Figure 15a shows the time course of the coefficient $a$ for the first two modes. The marked sections show both the course of periodic vortex shedding (Fig. 15b) and a short loss of vortexstreet stability (Fig. 15c).

Owing to the length of some records and the frequency of occurrence (in tens of seconds), the latter, for this event for individual settings and Reynolds numbers, has not been determined yet. The POD analysis also showed that it depends on how many frames are used for evaluation and whether the selected set includes this significant loss of stability. When processing data from the POD visualization, it is also important to recognize when the change in the POD coefficients is caused by the image quality and when it is influenced by vortex-street decay. Therefore, without prior knowledge on the visual record, it is not possible to determine the time course of the POD coefficient and when the anomaly occurs.

\subsubsection{Energy in POD modes}

As has been mentioned previously regarding POD, important phenomena and processes are contained in its first modes, as evidenced by the values of relative energies. Analyses show that the fewer disturbances in the system (Brevis and Garcia-Villalba 2011) refer to this as highly coherent flow), the higher the percentage of the total energy contained in the first two modes.

Figure 16 shows the relative energy for the single cylinder and each position from the visualizations for $D / d=1,1.67$, and 2.5 and $R e=500,1200$, and 2500 and for $D / d=2.5$ and $R e=500$ from PIV data. It is not easy to determine what exactly affects the relative energy in each position.
One of the assumptions in the evaluation was that the higher coherence (and fewer disorders) of the wake, the greater the difference between the relative energy value of the first two modes and the higher modes. However, this is not so clear from the POD results from the visualization shown in Fig. 16. Position 1 of the second cylinder does not significantly affect the wake, especially at higher $D / d$ values, which corresponds to the energy values of the first two modes in all configurations and for all Reynolds numbers. At position 2, the occurrence of disturbances in the observed area is more clearly shown. The ratio $D / d=1$ with a higher Reynolds number weakens the dominant structures, which is reflected in the decrease of the relative energy values against those in position 1 (Fig. 16b, c) and the increase of energy in higher modes. With increasing $D / d$ values, the vortex street of the first cylinder is not as weakened by the second cylinder, and the energy in this position approaches the value of position 1 . The rising Reynolds number has the same effect; for example, for $D / d=2.5$ and $R e=2500$, the relative energy for position 2 is higher than that for position 1 (Fig. 16i). The occurrence of disturbances in the wake of position 3 results in low relative energies in almost all cases, particularly in the small-value differences between the first and third or fourth POD modes. Interestingly, for $D / d=1$, this characteristic corresponds to that in position 2 . Further, it is clear that, with increasing Reynolds numbers, the disturbances slightly stabilize-emerging disturbances are suppressed and the differences between the first and third mode increase. A situation similar to that at position 3 also occurs at position 4 . Position 5 shows that the flow is more stable and the relative energy in most cases increases relative to position 4 . This is especially significant for higher $D / d$ ratios and higher Reynolds numbers. Although there are disturbances in position 6 owing to small misalignments of the second cylinder, the dominance of the structures is not affected much; therefore, this position has the highest relative energy values in the first two modes in almost all cases. However, it is surprising that these values are higher 
(a)

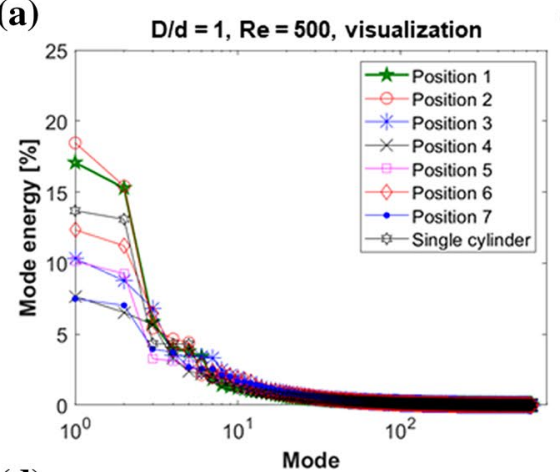

(d)

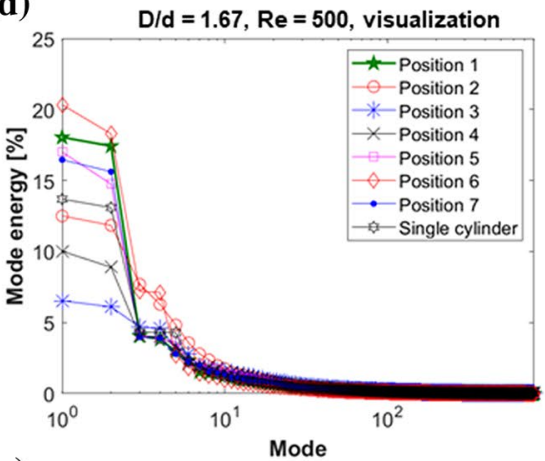

(g)

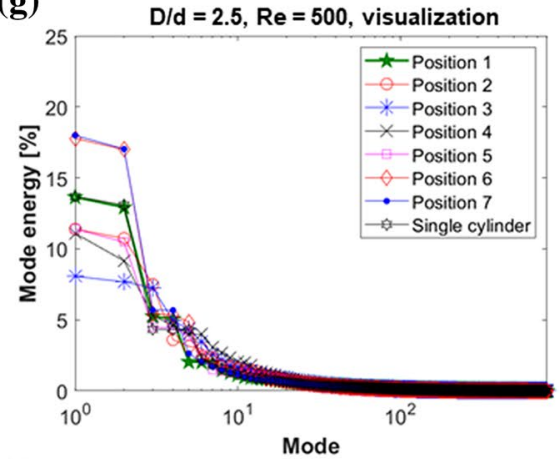

(j)

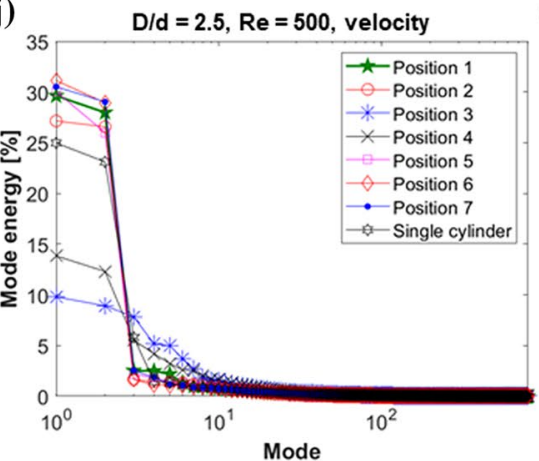

(b)

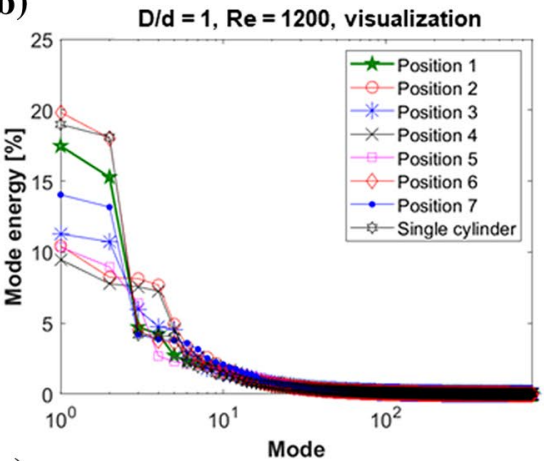

(e)

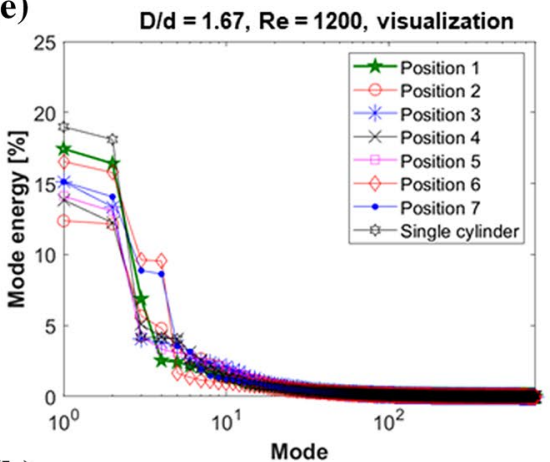

(h)

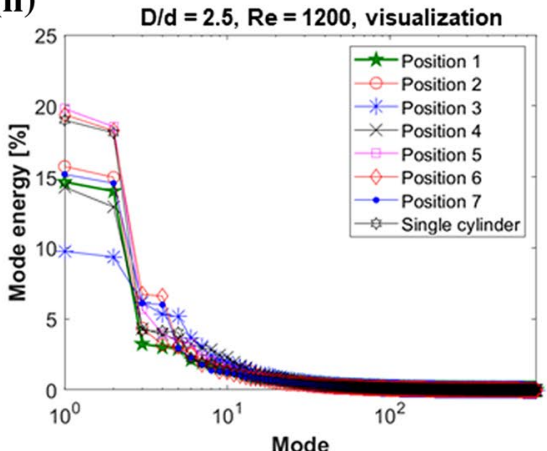

(k)

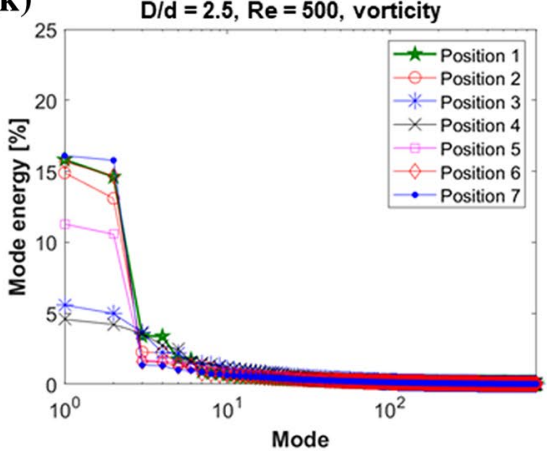

(c)

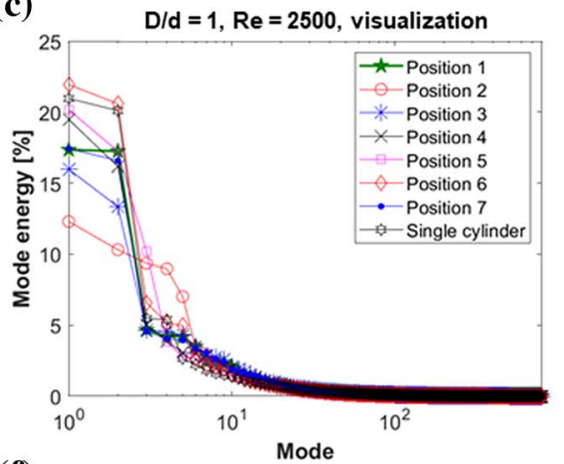

(f) $\quad \mathrm{D} / \mathrm{d}=1.67, \operatorname{Re}=2500$, visualization

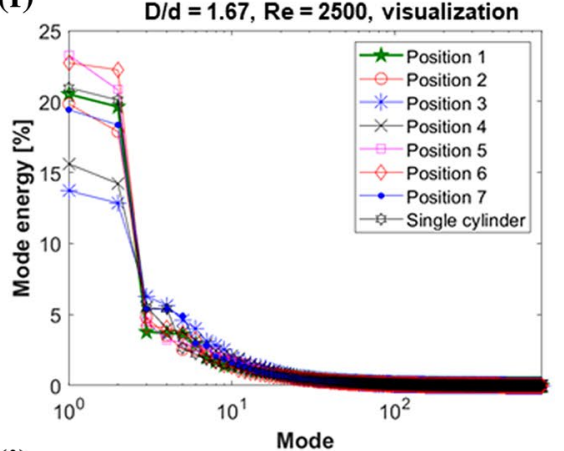

(i)
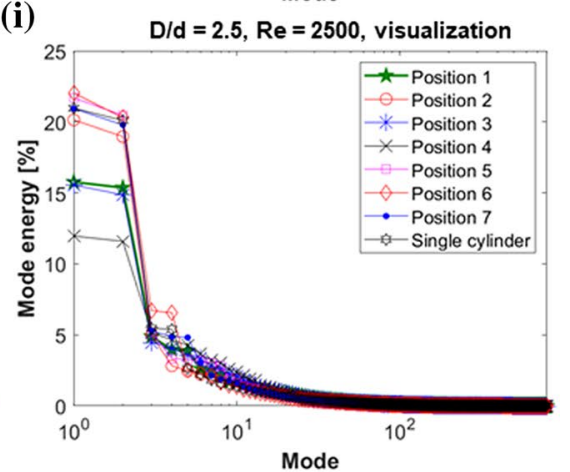

Fig. 16 Relative energy, a visualization, $D / d=1, R e=500$, b visualization, $D / d=1, \operatorname{Re}=1200$, (c) visualization, $D / d=1, R e=2500$, d visualization, $D / d=1.67, R e=500$, e visualization, $D / d=1.67$, $R e=1200, \mathbf{f}$ visualization, $D / d=1.67, R e=2500, \mathbf{g}$ visualization,
$D / d=2.5, R e=500, \mathbf{h}$ visualization, $D / d=2.5, R e=1200, \mathbf{i}$ visualization, $D / d=2.5, R e=2500$, $\mathbf{j}$ velocity field, $D / d=2.5, R e=500$, and $\mathbf{k}$ vorticity field, $D / d=2.5, R e=500$ than or equal to the relative energy values at position 7. As for the other observations, position 7 seems to be the most stable. There are several reasons for this. One of these may be the weakening of the strong structures in the flow, which results in a decrease in the value of the relative energy of the first two modes. 

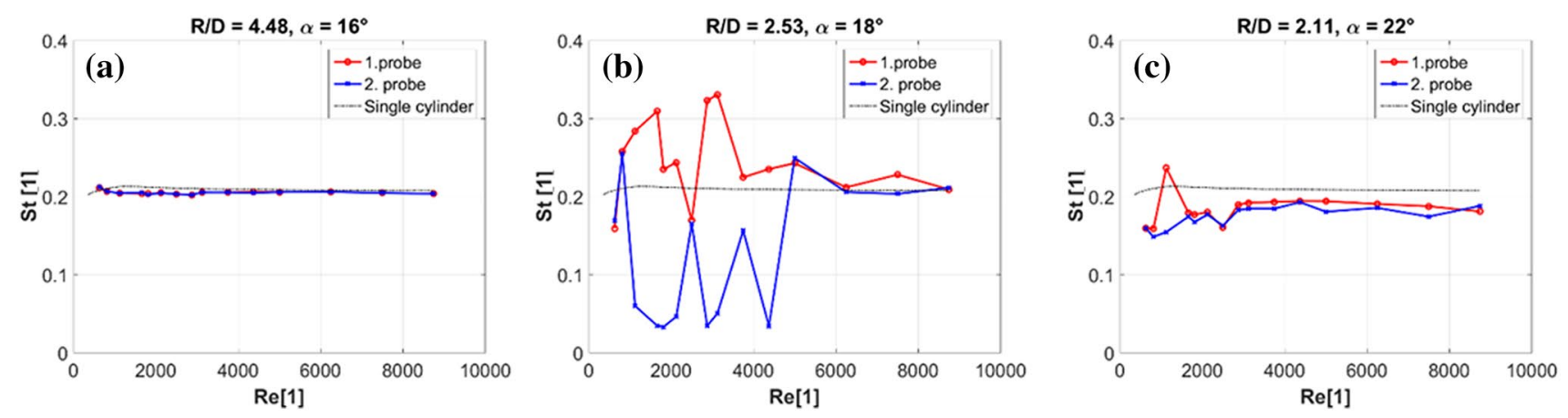

Fig. 17 St-Re dependence of CTA for two probes, a position 1, b position 3, and $\mathbf{c}$ position 4

Figure $16 \mathrm{j}$, k show the results of the energies obtained from PIV measurements. The kinetic energy values were determined from the velocity fields using the POD method (Fig. 16j) and the enstrophy values (Fig. 16k) were obtained from the fields of vorticity. The overall energy flow of both analyses is similar-they differ only at positions 4 and 5, where they have a lower relative value for enstrophy than kinetic energy. Another difference is the relative energy values themselves. POD velocity fields have higher values than POD vorticity fields.

As mentioned before, the percentages of relative energy show how important the structures are in terms of the studied complex. Higher percentages of kinetic energy may represent a stronger influence of translational movement, and smaller percentages of enstrophy indicate a greater presence of disorders in the wake. This is very evident from the value of enstrophy at positions where the most disturbances were recorded in the given setting $(D / d=2.5, R e=500)$, i.e., at positions 3 and 4 . In Fig. $16 \mathrm{j}$ at position 3 , the first mode has a relative kinetic energy value of $9.8 \%$; the second, $8.92 \%$; the third, $7.83 \%$; and the fourth, $5.17 \%$. These are very low values for the first two modes and very high values for the 3rd and 4th modes. The same applies for position 4. Measurement results from two probes can partly answer why. Figure 17 shows the measurement results for positions 1, 3, and 4. For positions 3 and 4, it is clear that the Strouhal numbers differ significantly, depending on the probe, compared to position 1. The greatest differences occur at lower Reynolds numbers. Therefore, it can be concluded that there is also a significant movement of structures in the direction of the z-axis, perpendicular to the observed plane. The values of the relative energies at positions 1, 6, and 7 show that there are not too many disturbances in the flow and it is, therefore, more coherent. At position 5, the lower value of relative enstrophy indicates the occurrence of some small disturbances, which is similar but weaker than at position 2 .

\section{Conclusion}

Data analysis using the POD method was applied to data from PIV measurements and data obtained from visualizations. Two types of data were used from the PIV-velocity field and vorticity field. The analysis of PODs obtained their flow patterns, which were related to the input quantity and their relative energy. The POD method applied to the velocity field in its modes exhibited the strongest moving structures, and the relative energy expressed here is kinetic energy (in the area). The first POD modes from the vorticity fields presented the strongest coherent structures and their relative energy-in this case, enstrophy. As for the visualization measurement, there is no physical quantity in the case of input data. The POD modes of visualizations showed significant structures and their relative energy by varying the gray intensity. An analogy to the POD modes of speed and vorticity was subsequently sought in these results. Other measurements and analyses contributed to the recognition of structures and their importance. Therefore, it was possible to identify the individual structures in the flow, to determine their importance, and to obtain an overall view of the flow field. From the calculated POD coefficients, the dominant frequencies of each mode were determined using frequency analysis; Strouhal numbers were calculated from these results. A comparison with CTA data revealed that the Strouhal numbers match in most cases. Where this was not the case, further analyses have shown that, in configurations where there is a higher occurrence of disorders, there is a noticeable change in the size of the dominant frequency, depending on the position of the hot-wire probe. The POD method does not analyze events in points but across an entire area; the first two modes are usually representative of the strongest structures, which is one of the ways in which this method differs from CTA. Another aspect of the POD is that, in some cases, the strongest structures in the first two modes 
differ slightly in their relative energy value against those in the following modes. Therefore, if this was the case in some configurations, it was pointed out that the determination of a single dominant frequency characterizing the investigated area in the wake was basically impossible, as confirmed by CTA data. From the analysis of the POD patterns using visualizations, it was shown how to interpret the structures contained in the first modes and identify likely coherent structures. From the presented results, it is clear that the POD method can significantly help in the identification of structures in the flow, not only from the input data-whose essence is the change of physical quantities-but also from data where only the visual characteristics change. Therefore, it is analogous to physical processes in the flow field.

Acknowledgements The author acknowledges the support received from the Centre of 3D volumetric velocimetry-COLA, supported by the European Union (CZ.2.16/3.1.00/21569) Operating Program Prague, and from the student grant competition SGS20/112/ OHK2/2T/12.

Open Access This article is licensed under a Creative Commons Attribution 4.0 International License, which permits use, sharing, adaptation, distribution and reproduction in any medium or format, as long as you give appropriate credit to the original author(s) and the source, provide a link to the Creative Commons licence, and indicate if changes were made. The images or other third party material in this article are included in the article's Creative Commons licence, unless indicated otherwise in a credit line to the material. If material is not included in the article's Creative Commons licence and your intended use is not permitted by statutory regulation or exceeds the permitted use, you will need to obtain permission directly from the copyright holder. To view a copy of this licence, visit http://creativecommons.org/licenses/by/4.0/.

\section{References}

Aubry N (1991) On the hidden beauty of the proper orthogonal decomposition. Theoret Comput Fluid Dyn 2(5-6):339-352. https://doi. org/10.1007/bf00271473

Berkooz G, Holmes P, Lumley JL (1993) The proper orthogonal decomposition in the analysis of turbulent flows. Annu Rev Fluid Mech 25(1):539-575

Breuer KS, Sirovich L (1991) The use of the Karhunen-Loève procedure for the calculation of linear eigenfunctions. J Comput Phys 96(2):277-296. https://doi.org/10.1016/0021-9991(91)90237-f

Brevis W, García-Villalba M (2011) Shallow-flow visualization analysis by proper orthogonal decomposition. J Hydraul Res 49(5):586-594. https://doi.org/10.1080/00221686.2011.585012

Chatterjee A (2000) An introduction to the proper orthogonal decomposition. Curr Sci 78(7):808-817

Choi H, Lee I, Jeon WP, Kim J (2008) Control of flow over a bluff body. Annu Rev Fluid Mech 40:113-139

Fey U, König M, Eckelmann H (1998) A new Strouhal-Reynoldsnumber relationship for the circular cylinder in the range $47<\operatorname{Re}<2 \times 10^{5}$. Phys Fluids $10(7): 1547-1549$. https://doi. org/10.1063/1.869675
Frederich O, Luchtenburg DM (2011) Modal analysis of complex turbulent flow. In: 7th International symposium on turbulence and shear flow phenomena (TSFP-7), Ottawa, Canada

Graftieaux L, Michard M, Grosjean N (2001) Combining PIV, POD and vortex identification algorithms for the study of unsteady turbulent swirling flows. Meas Sci Technol 12(9):1422-1429. https ://doi.org/10.1088/0957-0233/12/9/307

Graham MD, Kevrekidis IG (1996) Alternative approaches to the Karhunen-Loeve decomposition for model reduction and data analysis. Comput Chem Eng 20(5):495-506

Gurka R, Liberzon A, Hetsroni G (2006) POD of vorticity fields: a method for spatial characterization of coherent structures. Int J Heat Fluid Flow 27(3):416-423. https://doi.org/10.1016/j.ijhea tfluidflow.2006.01.001

Holmes P, Lumley J, Berkooz G, Rowley C (1996) Turbulence, coherent structures, dynamical systems and symmetry (Cambridge monographs on mechanics). Cambridge University Press, Cambridge. https://doi.org/10.1017/CBO9780511622700

Hu JC, Zhou Y (2008) Flow structure behind two staggered circular cylinders. Part 1. Downstream evolution and classification. J Fluid Mech 607:51-80

Hussain A (1986) Coherent structures and turbulence. J Fluid Mech 173:303-356. https://doi.org/10.1017/S0022112086001192

Hwang Y, Choi H (2006) Control of absolute instability by basic-flow modification in a parallel wake at low Reynolds number. J Fluid Mech 560:465-475

Hyhlik T, Zelezny P, Cizek J (2013) Visualization and modal decomposition of vortex street behind circular cylinder. Eur Phys J Web of Conf 45:01043. https://doi.org/10.1051/epjconf/20134501043

Ishigai S, Nishikawa E, Nishimura K, Cho K (1972) Experimental study on structure of gas flow in tube banks with tube axes normal to flow: Part 1, Karman vortex flow from two tubes at various spacings. Bull JSME 15(86):949-956. https://doi.org/10.1299/ jsme1958.15.949

Kevlahan NKR, Hunt JCR, Vassilicos JC (1994) A comparison of different analytical techniques for identifying structures in turbulence. Appl Sci Res 53(3-4):339-355. https://doi.org/10.1007/ bf00849109

Kostas J, Soria J, Chong MS (2005) A comparison between snapshot POD analysis of PIV velocity and vorticity data. Exp Fluids 38:146-160. https://doi.org/10.1007/s00348-004-0873-4

Le Clainche S, Li JI, Theofilis V, Soria J (2015) Flow around a hemisphere-cylinder at high angle of attack and low Reynolds number. Part II: POD and DMD applied to reduced domains. Aerosp Sci Technol 44:88-100. https://doi.org/10.1016/j.ast.2014.10.009

Lumley JL (1967) The structure of inhomogeneous turbulence. In: Yaglom AM, Tatarski VI (eds) Atmospheric turbulence and wave propagation. Nauka, Moscow, pp 166-178

Roshko A (1954) On the development of turbulent wakes from vortex streets. National advisory committee for aeronautics, Washington DC

Schmid PJ, Li L, Juniper MP, Pust O (2011) Applications of the dynamic mode decomposition. Theoret Comput Fluid Dyn 25:249-259. https://doi.org/10.1007/s00162-010-0203-9

Sirovich L (1987) Turbulence and the dynamics of coherent structures part I: Coherent structures. Q Appl Math 45(3):561-571

Strykowski PJ, Sreenivasan KR (1990) On the formation of vortex 'shedding' at low Reynolds numbers. J Fluid Mech 218:71-107

Sumner D, Price SJ, Païdoussis MP (2000) Flow-pattern identification for two staggered circular cylinders in cross-flow. J Fluid Mech 411:263-303. https://doi.org/10.1017/S0022112099008137

Tang SL, Djenidi L, Antonia RA, Zhou Y (2015) Comparison between velocity- and vorticity-based POD methods in a turbulent wake. Exp Fluids 56:169. https://doi.org/10.1007/s00348-015-2038-z 
Vitkovicova R, Yokoi Y (2019) The second frequencies in the wake behind two circular cylinders. Eur Phys J Web Conf 213:02093. https://doi.org/10.1051/epjconf/201921302093

Wang HF, Cao HL, Zhou Y (2014) POD analysis of a finite-length cylinder near wake. Exp Fluids 55:1790. https://doi.org/10.1007/ s00348-014-1790-9

Williamson CHK (1996) Vortex dynamics in the cylinder wake. Annu Rev Fluid Mech 28:477-539

Wong CW, Zhou Y, Alam MM (2014) Dependence of flow classification on the Reynolds number for a two-cylinder wake. J Fluids Struct 49:485-497

Yokoi Y, Vitkovicova R (2017) Experimental investigation of the mutual interference flow of two circular cylinders by flow visualization. Eur Phys J Web Conf 143:02146. https://doi.org/10.1051/ epjconf/201714302146

Zdravkovich MM (1977) Review of flow interference between two circular cylinders in various arrangements. ASME J Fluids Eng 99(4):618-633
Zdravkovich MM (1987) The effects of interference between circular cylinders in cross flow. J Fluids Struct 1(2):239-261. https://doi. org/10.1016/S0889-9746(87)90355-0

Zdravkovich MM (1997) Flow around circular cylinders. Oxford University Press, Oxford

Zhang Q, Liu Y, Wang S (2014) The identification of coherent structures using proper orthogonal decomposition and dynamic mode decomposition. J Fluids Struct 49:53-72. https://doi. org/10.1016/j.jfluidstructs.2014.04.002

Zhou Y, Feng SX, Alam MM, Bai HL (2009) Reynolds number effect on the wake of two staggered cylinders. Phys Fluids 21:125105

Publisher's Note Springer Nature remains neutral with regard to jurisdictional claims in published maps and institutional affiliations. 